NBSIR 84-3002

\title{
RESIDUAL-STRESS MEASUREMENTS USING SHEAR-HORIZONTAL WAVES FROM ELECTROMAGNETIC ACOUSTIC TRANSDUCERS
}

National Bureau of Standards U.S. Department of Commerce Boulder, Colorado 80303

March 1984 



\title{
RESIDUAL-STRESS MEASUREMENTS USING SHEAR-HORIZONTAL WAVES FROM ELECTROMAGNETIC ACOUSTIC TRANSDUCERS
}

\author{
R. B. King \\ C. M. Fortunko
}

Fracture and Deformation Division National Bureau of Standards

Boulder, Colorado 80303

March 1984

Funded by:

U.S. Navy, David Taylor Naval Ship Research and

Development Center, Annapolis, Maryland 21402

Office of Nondestructive Evaluation, National Bureau of Standards, Washington, D.C. 20234

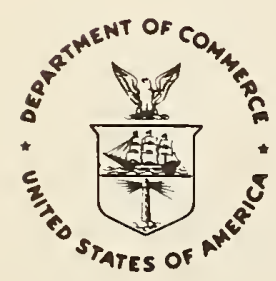

U.S. DEPARTMENT OF COMMERCE, Malcolm Baldrige, Secretary 

Residual-Stress Measurements Using Shear-Horizontal Waves from Electromagnetic

Acoustic Transducers

R. B. King* and C. M. Fortunko**

Fracture and Deformation Division

National Bureau of Standards

Boulder, Colorado 80303

This collection of technical papers covers a two-year effort on a novel approach to measuring residual stresses using changes in ultrasonic wave velocities of horizontally polarized shear waves produced by electromagnetic acoustic transducers. The initial three papers deal with the theoretical developments pointing to the measurement of in-plane residual stress as well as preliminary experimental verification. The later two papers generalize this theory and extend it to include surface residual stress measurement in an arbitrary plane; again, empirical validation is included.

Major support for this work came from the U.S. Navy, David Taylor Ship Research and Development Laboratory. Support also came from the Office of Nondestructive Evaluation of the National Bureau of Standards.

Key words: acoustic; electromagnetic; nondestructive; residual stress; shear horizontal; velocity.

*Present Address: IBM, 5600 Cottle Rd., San Jose, California, 95193

**Present Address: Aerojet Ordnance, 2521 Michelle Dr., Tustin, California, 92680 
In-Plane Residual-Stress Measurements (1982) ................ 1

1. Evaluation of Residual States of Stress and Material Texture Using Ultrasonic Velocity Measurements with Electromagnetic Acoustic Transducers, 1982 IEEE U1trasonics Symposium, ed. by B. R. McAvoy (IEEE, New York, 1982) pp. 885-888............ 3

2. Evaluation of Residual Stress States Using Horizontally Polarized Shear Waves, Review of Progress in Quantitative Nondestructive Evaluation, Vol. 2B, ed. by D. 0. Thompson and D. E. Chimenti (Plenum Press, New York, 1983) pp. 1327-

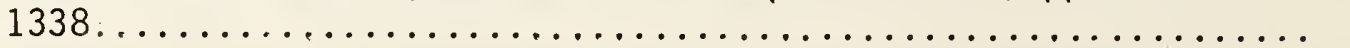

3. Determination of In-Plane Residual Stress States in Plates Using Horizontally Polarized Shear-Waves, Journal of Applied Physics, 54(6), pp. 3027-3035, 1983.................. 19

Arbitrary Plane Residual-Stress Measurements (1983)............ 29

4. Acoustoelastic Evaluation of Arbitrary Plane Residual Stress States in Nonhomogeneous, Anisotropic Plates, Ultrasonics,

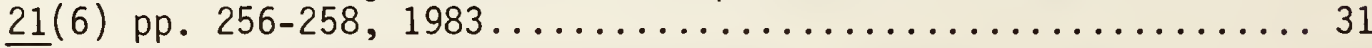

5. Evaluation of Surface Residual Stresses Using Horizontally Polarized Shear Waves at Grazing Incidence... 
IN-PLANE RESIDUAL-STRESS

MEASUREMENTS (1982) 

EVALUATION OF RESIDUAL STATES OF STRESS AND MATERIAL TEXTURE USING ULTRASONIC VELOCITY MEASUREMENTS WITH ELECTROMAGNETIC ACOUSTIC TRANSDUCERS

\author{
R. B. King and C. M. Fortunko
}

National Bureau of Standards

Fracture and Deformaticn Division

Boulder, Colorado 80303

In this paper a new approach for using acoustic measurements to evaluate residual stresses in the presence of unknown material property variation is presented. Procedures previously applied to the evaluation of stress with acoustic measurements are reviewed, and it is shown that these involve using measurements with bulk waves propagating along the normal to the surface of a plate and do not provide sufficient information to separate the influences of stress and material property variations. To overcome this fundamental limitation, an alternative theory is developed that governs the propagation of shear waves polarized horizontally with respect to the surface of a plate (SH-waves), but propagating at oblique angles with respect to the surface normal. The question of separating the effects of residual stress and material properties on acoustic velocity is addressed in detail. A practical experimental procedure is developed that permits the evaluation of the in-plane components of the principal stresses in a plate exhibiting an unknown inhomogeneous initial anisotropy caused by material texture or microstructure. The procedure is then verified experimentally using an aluminum specimen with a known residual stress state, but unknown initial anisotropy.

\section{Introduction}

In recent years considerable attention has been focused on the possibility of evaluating residual stress states in practical engineering materials by exploiting the fact that the presence of stress influences the velocities of elastic waves in a solid. However, progress has been slow because most practical engineering materials exhibit unknown material property variations, which also influence the elastic wave velocities. In practice, the problem can only be solved by developing a procedure permitting the unambiguous separation of the influences of the main factors determining the elastic wave velocities: metallurgical properties, stress, and temperature.

In this paper the problem of separating the effects of stress and metallurgical variations on the velocities of elastic waves in weakly anisotropic polycrystalline aggregates is addressed. Restricting measurements to elastic waves propagating along the normal to a plate does not provide sufficient information to separate the effects of stress from those of metallurgical variations because while several unknown stress components and material properties are present, only three independent measurements can be made. To overcome this limitation it is proposed that additional information be obtained from measurements of elastic wave velocities along directions that do not coincide with one of the principal stress directions. In particular, it is shown that sufficient information can be obtained using measurements of shear waves propagating in planes of symmetry of the anisotropic, polycrystalline aggregate.

To permit evaluation of stress and anisotropy from the velocity measurements, a special theory of acoustoelasticity is developed. A closed form theory is developed that describes the propagation of pure shear waves propagating in the planes of symmetry of the polycrystalline aggregate. Based on this theory, an experimental procedure is developed that permits the evaluation of biaxial stress states in plates when the principal stress directions lie in the planes of symmetry of the anisotropic, polycrystalline aggregate. The proposed experimenta procedure has important technological applications, particularly in the nondestructive evaluation of residual stresses in butt weldments.

The proposed experimental procedure is verified using an aluminum plate specimen containing a cylindrical plug that produces a known stress state. A new electromagnetic-acoustic transducer (EMAT) configuration is used that permits the generation and detection of horizontally polarized shear waves (SH-waves) from a very wide range of oblique propagation directions, and significantly increases the reproducibility of the measurements. The measured stress distributions are then compared with strain gauge data and calculated values.

Theory of Acoustoelasticity - Media of Orthorhombic

The theory of acoustoelasticity for media of orthorhombic symmetry can be developed from the equations of motion for a solid in the presence of stress. Following Tokuoka and Iwashimizu [1], the expression governing propagation of plane waves in a stressed anisotropic medium is found to be:

$\left[\tau_{i j k \ell} p_{i} p_{\ell}+\left(\sigma_{i \ell} p_{i} p_{\ell}-p v^{2}\right) \delta_{j k}\right] \sigma_{k}=0$

where $p_{j}$ is the direction of the wave normal, $d_{j}$ is the unit vector in the direction of particle motion, $\sigma_{j}$ is the stress tensor, $p$ is the density, $v$ is the "wabe phase speed, and $C_{i j}$ is a function of the second and third order 'elfstic tensors of the material, $C_{i j k \ell}$ and $C_{i j k \ell m n}$. 
Closed form solutions of the eigenvalue problem of Eq. l are obtainable when the propagation direction lies in a plane of material symmetry. The details of the derivation are presented in Ref. [2]. The key results for weakly anisotropic media are:

$\left.\frac{\mathrm{SH}_{23}-\mathrm{SH}_{13}}{S H^{0}}\right|_{\phi}=\frac{\cos ^{2} \phi}{2 p\left(S H^{0}\right)^{2}}\left(C_{55}-C_{44}\right)+a(\phi)\left(\sigma_{2}-\sigma_{1}\right)$

and

$\left.\frac{S H_{23}+S H_{13}-2 S H^{0}}{2 S H^{\circ}}\right|_{\phi}=b(\phi)\left(\sigma_{2}+\sigma_{1}\right)$

where $\mathrm{SH}_{i j}$ denotes the velocity of an SH-wave propagatint in the $X_{j}-X_{j}$ plane, $\sigma_{2}$ and $\sigma_{3}$ are the in-plane principal stress components, $a(\phi)$ and $b(\phi)$ are functions of components of the second and third order elastic tensors, and $\mathrm{SH}^{\circ}$ denotes the mean unstressed velocity, which is explicitly giver, by:

$$
\begin{aligned}
\mathrm{SH}^{\circ}= & \frac{1}{2}\left[\left(\frac{C_{44} \cos ^{2} \phi+C_{66} \sin ^{2} \phi}{\rho_{0}}\right){ }^{1 / 2}\right. \\
& \left.+\left(\frac{C_{55} \cos ^{2} \phi+C_{66} \sin ^{2} \phi}{\rho_{0}}\right)^{1 / 2}\right]
\end{aligned}
$$

An estimate of $\mathrm{SH}^{\circ}$ may be used in the denominator of Eqs. 2 and 3 , because a small error in the denominat results in a higher order error in the calculated stress states. However, the exact value of $\mathrm{SH}^{\circ}$ is needed in the numerator of Eq. 3 because a small error in the numerator results in a large error in the calculated stress states.

The importance of Eqs. 2 and 3 is that the same unknowns are present regardless of the value of $\$$. As a consequence, measurements at different values of $\phi$ provide additional information without increasing the number of unknowns. Equation 2 is particularly important because only two unknowns are present: $\left(C_{55}\right.$ - $\left.C_{44}\right)$ and $\left(\sigma_{2}-\sigma_{1}\right)$. Thus, measurements at only two values of are required to evaluate the quantity $\left(\sigma_{2}-\sigma_{1}\right)$.

\section{An Experimental Procedure}

It follows from the preceding discussion that the residual stress state and the relevant elastic constants can be uniquely determined by making precise velocity measurements with off-axis SH-waves in the planes of material symmetry. To separate the effects of texture and stress, measurements must be made in mutually orthogonal material symmetry planes.

It is straightforward to determine the difference between two principal stresses by making relative measurements and using Eq. 2. In this case, the procedure involves first making a velocity measurement of an SH-wave propagating in the $x_{2}-x_{3}$ plane at an angle $\phi$ with respect to $x_{3}$ axis. Next, the measurement is repeated in the $x_{1}-x_{3}^{3}$ plane for the same angle $\phi$. Both measurements must then be repeated for a different value of $\phi$. The difference between the two measurements is then used in $E_{q} .2$ to evaluate $\left(\mathrm{O}_{2}-\sigma_{1}\right)$ and the quantity $\left(\mathrm{C}_{55}-\mathrm{C}_{44}\right)$. (In many technological applications the knowledge of $\left(\sigma_{2}-\sigma_{1}\right)$ is sufficient because additional informatio refated to the specimen geometry is usually available to estimate the relative magnitudes of $\sigma_{2}$ and $\sigma_{1}$. )

In principle, the separate values of $\sigma_{2}$ and $c_{1}$ can be determined experimentally by using Eqs. 2 and 3. However, in this case a set of five independent measurements must be performed at different values of $\phi$. The five measurements are needed to determine the values of $\sigma_{1}, \sigma_{2}, C_{44}, C_{55}$, and $C_{66}$. In practice, an additional measufement may be required to compensate for any path length variations.

\section{A Practical Experimental Configuration}

To obtain experimental data for utilizing Eqs. 2 and 3 , transducers of SH-waves are required that produce ultrasonic beam patterns with wide angular distributions. An electromagnetic acoustic transducer which meets this requirement is described in Ref. [2]. A representative ultrasonic radiation pattern of this EMAT is shown in Fig. 1. In Fig. 1, the transmitted ultrasonic amplitude (at $520 \mathrm{kHz}$ ) is plotted as a function of the angles $(\phi)$ in the plane normal to the surface of a plate and bisecting the transducer between the magnets (the sagittal plane). It is evident that the radiation pattern is very broad and, in particular, does not vanish at any angle in the sagittal plane. By reciprocity, the same directivity pattern applies upon reception. This property of the transducer is needed to study the velocities of ultrasonic waves as a function of propagation direction.

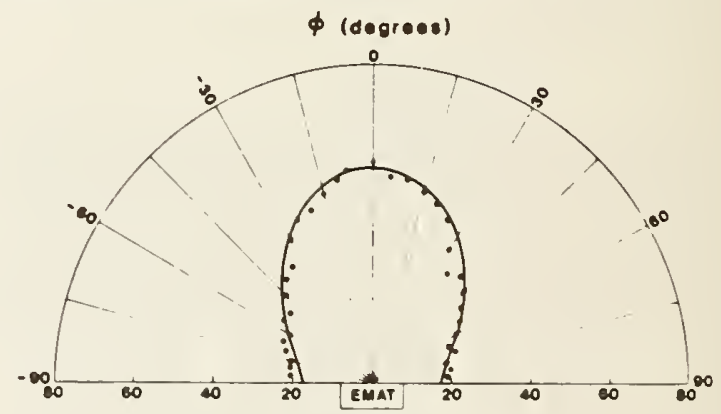

SIGNAL (mV)

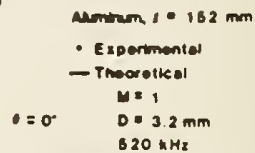

Figure 1. Ultrasonic radiation pattern of electromagnetic-acoustic transducer.

A practical experimental configuration for determining stress states in anisotropic plates is shown in Fig. 2. In the configuration of Fig. 2 two SH-wave EMATs are used. One of the EMATs is used as a generator and the other as a receiver of the probing ultraisonic signals. Because the two EMATs are aligned along the same sagittal plane, the receiver EMAT is sensitive only to ultrasonic 
signals with a displacement component parallel to the surfaces of the plate and normal to the sagittal plane (SH-waves).

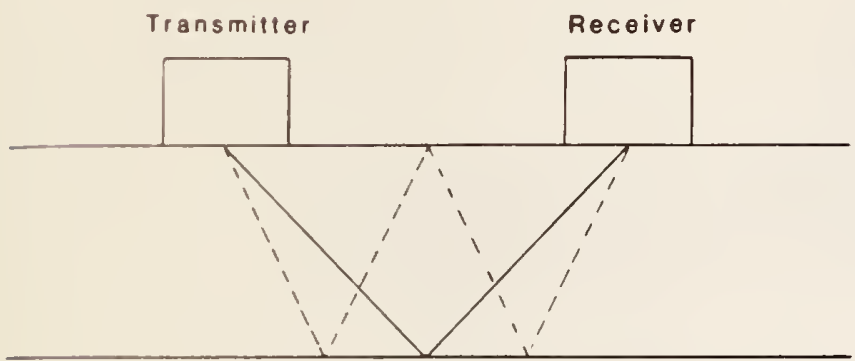

Figure 2. Transducer configuration for stress measurement.

In the special case when one of the principal stress directions is parallel to a crystal axis and the sagittal plane is also a plane of crystal symmetry, the received signals are pure $\mathrm{SH}$-waves. Since $\mathrm{SH}$-waves are decoupled from longitudinal (L) and vertically polarized shear (SV) waves upon reflection, they are not phase shifted upon reflection from the surfaces of the plate. This important observation results in a significant simplification in the inversion of the experimental data and forms the basis for the experimental determination of residual stress states in planar geometries.

An examination of $\mathrm{Fig} .2$ reveals that many independent ultrasonic signal paths (rays) exist that connect the centers of the generator and receiver EMATs. In fact, useful experimental information is available over a range of angles $\phi$, from grazing ( $\phi=$ $\left.90^{\circ}\right)$ to nearly normal $\left(\phi \sim 0^{\circ}\right)$. As a consequence, by measuring the phases of a number of the ultrasonic signals in planes of symmetry, sufficient information is provided to permit the unambiguous determination of the in-plane stress components, elastic constants and adjustment for plate thickness variations, if needed.

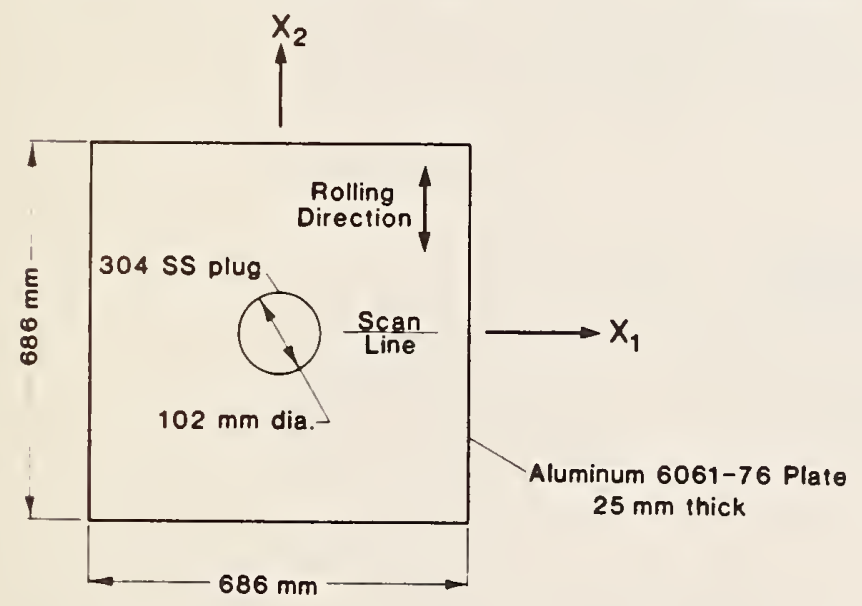

Figure 3. Specimen containing a known residual stress state.

\section{Experimental Verfication}

To verify the experimental procedure, measurements were carried out on an aluminum plate specimen, 25-mm thick, which contained a known biaxial stress state and unknown initial anisotropy. Aluminum was chosen for this study because it is typical of engineering materials that although nominally elastically isotropic, exhibit a small amount of anisotropy. The anisotropy causes an effect on velocity that is not negligible in comparison to the effect of stress. The design of the experimental specimen is shown in Fig. 3. The test plate was square, $686 \mathrm{~mm}$ on each side, and made of type 6061-T6 aluminum. In the center of the plate is a hole into which was inserted a circular plug, $102 \mathrm{~mm}$ in diameter, made of type 304 stainless steel. Prior to insertion, the stainless steel plug was cooled to $77 \mathrm{~K}$ and the aluminum test plate heated to $423 \mathrm{~K}$. After insertion, the resulting strains were monitored with strain gauges placed along radial lines until the whole test specimen was returned to the ambient temperature. The strain gage readings confirmed the theoretically predicted stress distribution.

To obtain ultrasonic data, the specimen shown in Fig. 3 was scanned using the experimental SH-wave EMAT configuration, as shown in Fig. 2. The scanning was carried out along one of the radial lines that coincide with the axis of plate symmetry, as shown in Fig. 3. To measure the phases of the ultrasonic signals, a modified system of Kino et al. [3] was used.

The relative velocity differences $\left(\mathrm{SH}_{23}-\mathrm{SH}_{3}\right) / \mathrm{SH}$ were calculated for several positions along the ${ }^{3}$ can line, with the $x_{2}$ axis taken to coincide with the plate rolling difection. The ultrasonic data were obtained from the first and third arrival signals, as shown in Fig. 2, with the SH-wave EMAT pair aimed along the scan line and, then, at $90^{\circ}$ with respect to the scan line. The first and third arrivals were selected because of the large difference in the angle defined by their rays and the plate surface normal, $33.8^{\circ}$ and $12.6^{\circ}$. The difference in the principal stresses, $\sigma_{2}-\sigma_{1}$, was then evaluated from the velocity measurements dsing Eq. 2. As discussed

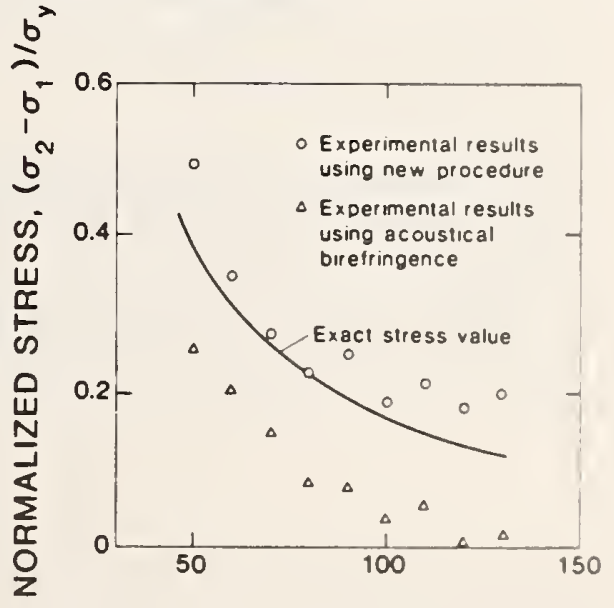

DISTANCE FROM PLUG BOUNDARY, $\mathrm{mm}$

Fiaure 4. Princioal stress difference evaluated in soecimen of figure 3 
in the preceding section, the constant $c(\phi)$ was neglected because of the weak anisotropy. The values of the constants $a\left(\phi_{1}\right)$ and $a\left(\phi_{2}\right)$ were determined independently using tensile cafibration specimens obtained from the ends of the test-block shown in Fig. 3. The calibration values determined were $\mathrm{a}\left(33.8^{\circ}\right)=3.7 \times 10^{-5} / \mathrm{MPa}, \mathrm{a}\left(12.6^{\circ}\right)=4.4 \times 10^{-5} / \mathrm{MPa}$. The calibration constants were found to vary with position, but the variations were less than 1\%. The experimental results for the ultrasonically measured values of $\sigma_{2}-\sigma$ are shown in Fig. 4. For comparison, the calculated values of $\left(\sigma_{2}-\sigma_{1}\right)$ from the elasticity solution are also shown in Fig. 4 . The calculated distribution is parabolic, and the experimental results are seen to follow this shape.

\section{Summary}

Closed-form expressions relating the velocities of elastic waves propagating at arbitrary angles in the planes of symmetry of weakly anisotropic, polycrystalline solids have been presented. In particular, it has been shown that using measurements of the velocities of pure shear waves as a function of propagation, direction, sufficient information is obtained to permit the evaluation of residual stress states in a practical plate geometry. In fact, measurements at only two independent propagation directions are required to obtain the difference in the two in-plane principal stress components. Furthermore, an experimental procedure, based on the new theory, has been proposed and evaluated using a new $\mathrm{SH}$-wave electromagnetic-acoustic transducer (EMAT) design.

\section{Acknowledgments}

The authors are indebted to $S$. A. Padget, J. Arvidson, Jr., and R. A. Schramm, who participated in the construction of the experimental apparatus and in the experiments described. The authors are also indebted to J. D. McColskey who prepared the specimens used in the experiments. This work was supported in part by the U.S. Navy, David Taylor Naval Ship Research and Development Center, and the Office of Nondestructive Evaluation of the National Bureau of Standards.

\section{References}

1. T. Tokuoka and Y. Iwashimizu, Int. J. Solids and Structures 9, 383 (1968).

2. R. B. King and C. M. Fortunko, "Determination of In-Plane Residual Stress States in Plates Using Horizontally Polarized Shear Waves", submitted to Journal of Applied Physics.

3. G. S. Kino, J. B. Hunter, G. C. Johnson, A. R. Selfridge, D. M. Barnett, G. Hermann, and C. R. Steele, J. Appl. Phys. 50, 2607 (1979). 
EVALUATION OF RESIDUAL STRESS STATES USING

HORIZONTALLY POLARIZED SHEAR WAVES

R. B. King and C. M. Fortunko

Fracture and Deformation Division

National Bureau of Standards

Boulder, Colorado 80303

\section{ABSTRACT}

A new approach for using acoustic measurements to evaluate residual stresses in the presence of unknown material property variations is presented. It is shown that measurements using shear waves propagating along the normal to the surface of a plate do not provide sufficient information to separate the influences of stress and material property variations. To overcome this fundamental limitation, an alternative theory is developed that governs the propagation of shear waves polarized horizontally with respect to the surface of a plate (SH-waves), but propagating at oblique angles with respect to the surface normal. The question of separating the effects of residual stress and material properties on acoustic velocity is addressed in detail. In addition, a practical experimental procedure is developed that permits the evalution of the in-plane components of the principal stresses in a plate exhibiting an unknown inhomogeneous inftial anistropy caused by material texture or microstructure. The procedure is then verified experimentally using an aluminum specimen with a known residual stress state, but unknown initial anisotropy.

\section{INTRODUCTION}

The application of acoustic velocity measurements to the evaluation of residual stresses has received considerable attention in recent years. A major problem has been the fact that practical engineering alloys exhibit considerable material property variations. These variations affect acoustic velocity and may mask the effects of stress. 
In this paper, the problem of evaluating residual stresses from velocity measurements in alloys with unknown materlal property variations is addressed. In particular, it is demonstrated that experimental procedures, in which velocity measurements of acoustic waves at normal incidence to the surface of a solid are made, do not provide sufficient information to determine the stresses unambiguously. Specifically, it is shown that the commonly used technique based on acoustical birefringence is hampered by the influence of material anisotropy.

A new technique is proposed which is based on measurements with horizontally polarized shear waves (SH-waves) propagating in a plate at arbitrary angles to the normal to the free surfaces. The new technique is a generalization of the acoustical birefringence method. It is shown theoretically that the technique provides enough information to permit the evaluation of in-plane stress components in the presence of unknown material anisotropy. An experimental procedure is then described that uses electromagneticacoustic-transducers (EMATs) to generate the probing SH-wave signals. Results of a verification experiment using a plate specimen containing a known residual stress state are presented.

\section{THEORY}

The basis for evaluation of stress with measurements of acoustic velocity is provided by the theory of acoustoelasticity, which relates changes in the velocity of elastic waves to the stress state in a solid. The most common application involves the use of bulk waves propagating at normal incidence to the surface of a plate. The velocity measurements with these waves are used to characterize the average through-thickness values of the in-plane stress components. Alloys are polycrystalline aggregates which can exhibit macroscopic anisotropic elastic properties due to microstructure or texture. The polycrystalline material is elastically equivalent to an anisotropic crystal. The acoustoelasticity relations for waves propagating along the normal to a plate made of anisotropic material are:

$$
\begin{aligned}
& \frac{v_{\ell}-v_{\ell}^{0}}{v_{\ell}^{0}}=A\left(\sigma_{1}+\sigma_{2}\right)+D\left(\sigma_{2}-\sigma_{1}\right) \\
& \frac{v_{S 2}-v_{S 1}}{v_{S}^{0}}=\frac{v_{S 2}^{0}-v_{S 1}^{0}}{v_{S}^{0}}+B\left(\sigma_{2}-\sigma_{1}\right)+E\left(\sigma_{1}+\sigma_{2}\right) \\
& \frac{v_{S 2}+v_{S 1}-2 v_{S}^{0}}{2 v_{S}^{0}}=C\left(\sigma_{1}+\sigma_{2}\right)+F\left(\sigma_{2}-\sigma_{1}\right)
\end{aligned}
$$


where $V_{\ell}, V_{S 2}$ and $V_{S 1}$ are the velocities in the presence of stress of a longitudinal wave, a shear wave polarized parallel to the $\mathrm{X}_{2}$ direction, and a shear wave polarized parallel to the $x$ direction, respectively; $V_{l}{ }^{\circ}$ is the velocity of a longitudinal wave in the unstressed state, $v_{S}{ }^{\circ}$ is the average shear wave velocity in the unstressed state, and $\sigma_{2}$ and $\sigma_{1}$ are the in-plane principal stress components. The constants $A$ through $F$ in Eqs. (1-3) are functionally dependent on the second and third order elastic constants of the solid, and are commonly referred to as acoustoelastic constants or stress-acoustic constants. It is shown that for solids with weak anisotropy and with principal directions of stress parallel to crystal axes, the constants $D, E$, and $F$ are small and may be neglected for practical measurement purposes.

Equations (1-3) have been applied in three ways. In the acoustical birefringence method, Eq. (2) is employed to determine the difference of the principal stresses from the relative difference of the velocities of shear waves polarized in the mutually orthogonal principal directions. ${ }^{2-4}$ Longitudinal wave measurements have been used by Kino and co-workers 5,6 to evaluate the quantity $\left(\sigma_{2}+\sigma_{1}\right)$ using Eq. (1). Guscha, et al. and Guz, et al, ${ }^{7,8}$ have used both shear and longitudinal wave measurements in an attempt to determine the separate values of $\sigma_{1}$ and $\sigma_{2}$ in nominally isotropic steels.

It is relatively straightforward to apply acoustic measurements to the characterization of applied stress states if independent measurements can be made in the unstressed state for in this case, the values of $\mathrm{V}_{\ell}^{\circ}, \mathrm{V}_{\mathrm{S} 1}{ }^{\circ}$, and $\mathrm{V}_{\mathrm{S}}{ }^{\circ}$ can be measured throughout the region of interest. S1 Hower, $^{S 2}$ the evaluation of residual stress states using acoustic velocity measurements is serfously complicated by the fact that constants characteristic of the unstressed state are not avallable. The nominal value of the velocities in the absence of stress can be measured using a separate reference sample of the same materfals. However, most alloys of engineering importance are not perfectly homogeneous. As a consequence, variations in the velocities in the unstressed state can be present that are of the same order of magnitude as the velocity variations caused by stress. In particular, in the comonly used acoustical birefringence method, the presence of initial anisotropy can result in an unknown, and not negligible, value of the quantity $\left(\mathrm{V}_{S 2}{ }^{\circ}-\mathrm{V}_{S}{ }^{\circ}\right) / \mathrm{V}_{\mathrm{S}}{ }^{\circ}$, even in nominally isotropic
materials. The importance of widely recognized. Various procedures have been proposed for providing information using other measurements in addition to acoustic velocity measurements to permit evaluation of stress in the presence of the unknown initial velocity term. 9 However, none of these procedures has been satisfactorily experimentally verifled to date. 
In this paper, a new technique is proposed for overcoming the problem of unknown initial anisotropy. The conventional birefringence method is limited by the fact that, with measurements confined to elastic waves propagating along the normal to the surface of a plate, insufficient data is obtained to separate the effects of stress and anisotropy. Equation (2) involves two unknowns, $\left(\mathrm{V}_{\mathrm{S} 2}{ }^{\circ}-\mathrm{V}_{\mathrm{S} 1}{ }^{\circ}\right) / \mathrm{V}_{\mathrm{S}}{ }^{\circ}$ and $\left(\sigma_{2}-\sigma_{1}\right)$, but only one quantity, $\left(\mathrm{V}_{\mathrm{S} 2}-\mathrm{V}_{\mathrm{S} 1}\right) / \mathrm{V}_{\mathrm{S}}{ }^{\circ}$, is measured. SAdditional information would in principle be provided by measuring the velocity of waves propagating at an angle with respect to the plate normal, but in general this approach would be difficult because a closed-form theory relating velocity to stress is not available and the waves are not pure modes. However, it is shown that for the special case of horizontally polarized shear waves (SH-waves) propagating in planes of crystal symmetry, in solids whose directions of principal stress are parallel to crystal axes, a closed-form theory can be derived. In particular, the following equation is shown to be valid for weakly anisotropic solids:

$$
\left.\frac{\mathrm{SH}_{23}-\mathrm{SH}_{13}}{\mathrm{SH}^{\circ}}\right|_{\theta}=\cos ^{2} \theta\left(\frac{\mathrm{C}_{55}-\mathrm{C}_{44}}{\mathrm{C}_{44}}\right)+a(\theta)\left(\sigma_{2}-\sigma_{1}\right)
$$

where $\mathrm{SH}_{23}$ and $\mathrm{SH}_{13}$ are the velocities of SH-waves propagating in the $X_{2}-X_{3}^{23}$ and $x_{1}-X_{3}^{3}$ planes of a plate, $X_{3}$ is parallel to the normal to the plate, $X_{1}$ and $X_{2}$ are parallel to crystal axes, $\theta$ is the angle between the propagation direction and the plate normal. $C_{55}$ and $C_{44}$ are elastic constants, $\mathrm{SH}^{\circ}$ is the average velocity in the unstressed state, given by

$$
\mathrm{SH}^{\circ}=\frac{1}{2}\left(\mathrm{SH}_{23}^{\circ}+\mathrm{SH}_{13}^{\circ}\right)
$$

The acoustoelastic constant $a(\theta)$ is a function of the second- and third-order elastic constants of the material.

The first term on the right-hand-side of Eq. (4) is equal to

$$
\left.\left(\frac{\mathrm{SH}_{23^{\circ}}-\mathrm{SH}_{13}^{\circ}}{\mathrm{SH}^{\circ}}\right)\right|_{\theta^{\circ}}
$$

Thus, Eq. (4) is a generalization of the usual acoustical birefringence relation, Eq. (2) and, in fact, reduces to that relation for the special case $\theta=0^{\circ}$. The main advantage of using Eq. (4) is that measurements can be made at various values of the angle $\theta$, thereby providing additional information without increasing the number of unknowns. Measurements at two values of $\theta$ provide two equations, which can be solved for the unknowns $\left(\mathrm{C}_{55}-\mathrm{C}_{44}\right) / \mathrm{C}_{44}$ and $\left(\sigma_{2}-\sigma_{1}\right)$. (Measurements at more than two angles of ${ }^{5}$ w 411 p $40 d u c e$ an overdetermined system of equations, which can be solved using least squares fitting.) 


\section{EXPERIMENTAL PROCEDURE}

To permit 1mplementation of Eq. (4), transducers capable of generating SH-waves propagating at arbitrary angles with respect to the plate normal are required. A practical electromagnetic-acoustic-transducer (EMAT) capable of fulfilling this requirement has been developed. A schematic representation of this transducer is shown in Fig. 1. A typical acoustic radiation pattern for the transducer in Fig. 1 is shown in Fig. 2: This pattern can be regarded as an angular spectrum of plane waves. Thus, by properly locating transmitting and recelving transducers appropriate rays representing plane waves at desired angles may be utilized. A practical experimental configuration for applying the transducer of F1g. 1 to stress characterization is shown in F1g. 3. The transmitting transducer and recelving transducer are aligned so that the plane passing between the magnets of the transducer and normal to the plate (the sagittal plane) is shared by the two transducers. SH-waves are generated by the transmitter which propagate in this plane and whose polarization direction is normal to 1t. Upon examining Fig. 3, it is evident that because of multiple reflections from the back surface of the plate, many rays connect the two transducers. Measurements with plane waves at various angles with respect to the plate normal thus can be made by utilizing different rays. The procedure for implementing the configuration in Fig. 4 is as follows. In a plate for which the crystal axes are known (e.g., a rolled plate) the transducers are oriented so that the sagittal plane is parallel to a crystal axis. A single echo is isolated by time-gating so that velocity of a plane wave at an angle $\theta$, with respect to the plate normal can be measured. The transducers are now rotated $90^{\circ}$ and the velocity measurement is repeated. The quantity

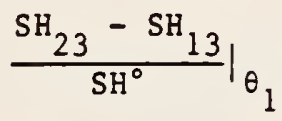

is now determined. This procedure is then repeated using a different echo, so that the relative velocity of plane waves propagating at a different angle, $\theta$, is now measured. Two equations are then avallable for evaluating the unknowns

$$
\left(\frac{C_{55}-C_{44}}{C_{44}}\right) \text { and }\left(\sigma_{2}-\sigma_{1}\right) \text {. }
$$

A precision velocity measuring system is required to implement the experimental procedure described. Since only relative measurements are needed to apply Eq. (4), a modifled version of the systet developed by Kino et al. 6 was utilized in the present work. In this system, the relative frequency change required to maintain a 


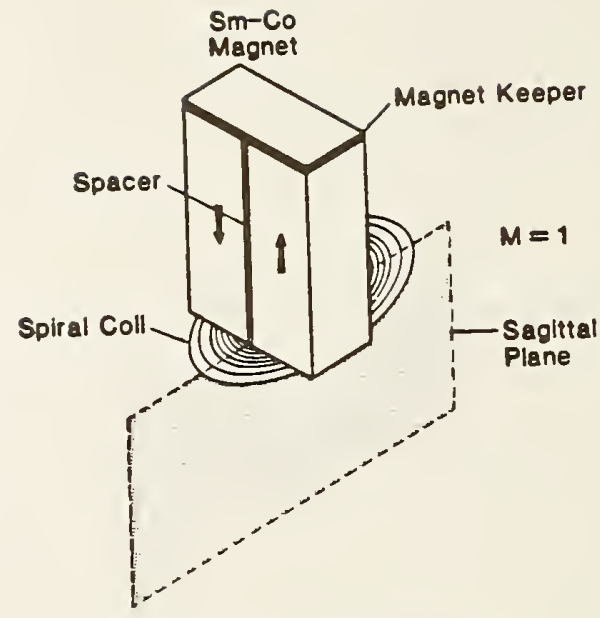

Fig. 1. A practical electromagnetic-acoustic transducer design.

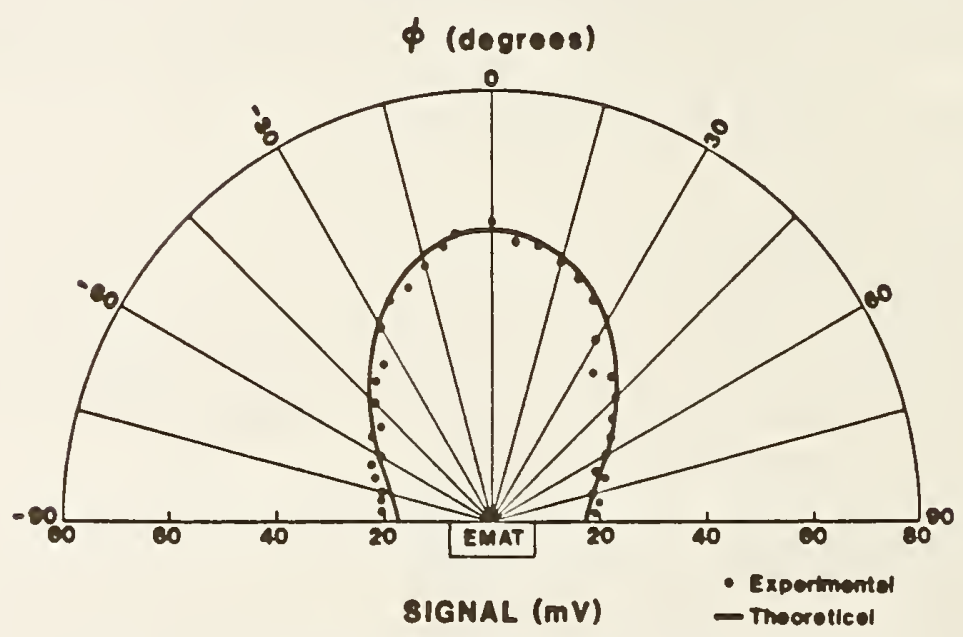

Fig. 2. Ultrasonic radiation pattern of EMAT. 


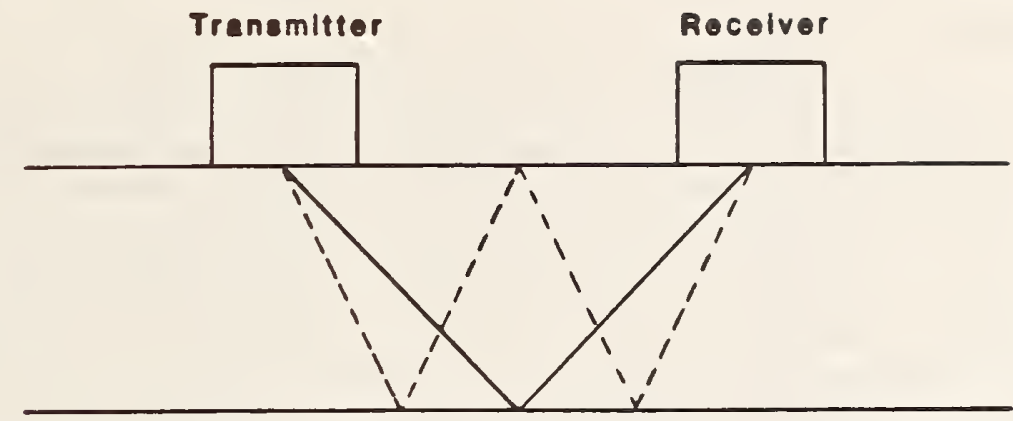

Fig. 3. Transducer configuration for stress measurement.

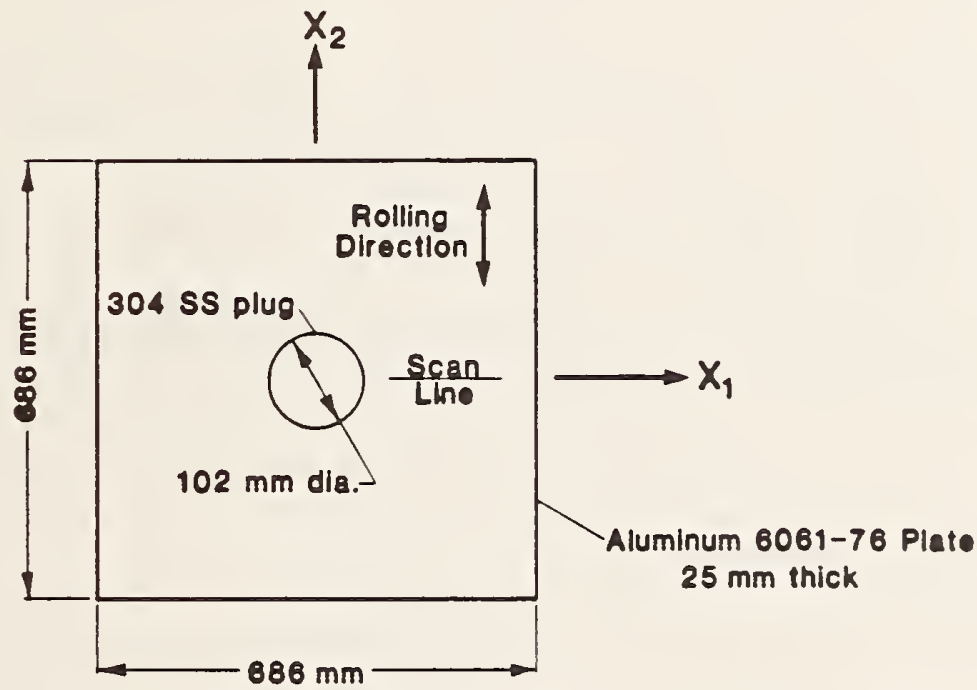

Fig. 4. Specimen containing a known residual stress-state.

constant phase difference between an acoustic signal and a reference is measured. Relative velocity changes can be evaluated using the relation

$$
\frac{\Delta V}{V}=\frac{\Delta f}{f}+\frac{\Delta l}{\ell}
$$

where $f$ denotes frequency and $\ell$ denotes acoustic path length. In using this system for making velocity measurements on two orthogonal planes as described above, the path length remains unchanged so that the term $\Delta \ell / \ell$ vanishes. 


\section{EXPERIMENTAL RESULTS}

To verify the theory and experimental procedure presented above, measurements were carried out on an aluminum plate specimen, 25 mm thick, which contained a known, biaxial, residual stress state and unknown initial anisotropy. The specimen design is shown in Fig. 4. A $100 \mathrm{~mm}$ hole was machined in the aluminum plate. The plate was heated to $423 \mathrm{~K}$ and an oversized plug of 304 stainless steel which had been cooled to $77 \mathrm{~K}$ was inserted in the hole. Strain gages had been placed on the aluminum plate so that the resulting residual stress state could be evaluated after the specimen returned to ambient temperature. The measured strains agreed with the predictions of an exact theory for this specimen. 10 The resulting values of $\left(\sigma_{2}-\sigma_{1}\right)$ as a function of position along one of the plate symmetry axes (normal to an edge) are shown in Fig. 5.

Before the ultrasonic experimental procedure described above could be used to evaluate the residual stresses in the specimen, the acoustoelastic effect had to be calibrated to determine the constants $a(\theta)$ in Eq. (4). Two values of $\theta$ were chosen, $33.8^{\circ}$ and $12.6^{\circ}$. These correspond to the first and third echoes from the back surface of the plate, respectively. A calibration specimen in the form of a tensile bar was cut from the end of the aluminum plate from which the specimen containing residual stress was made. The tensile axis was parallel to the rolling direction. Tension

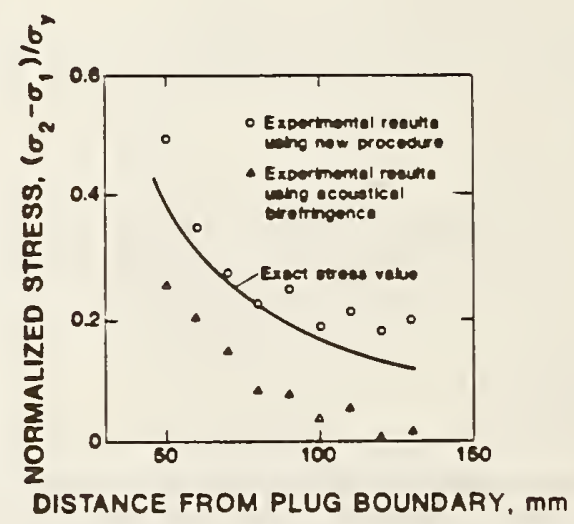

Fig. 5. Principal stress difference evaluated in specimen of Fig. 4. 


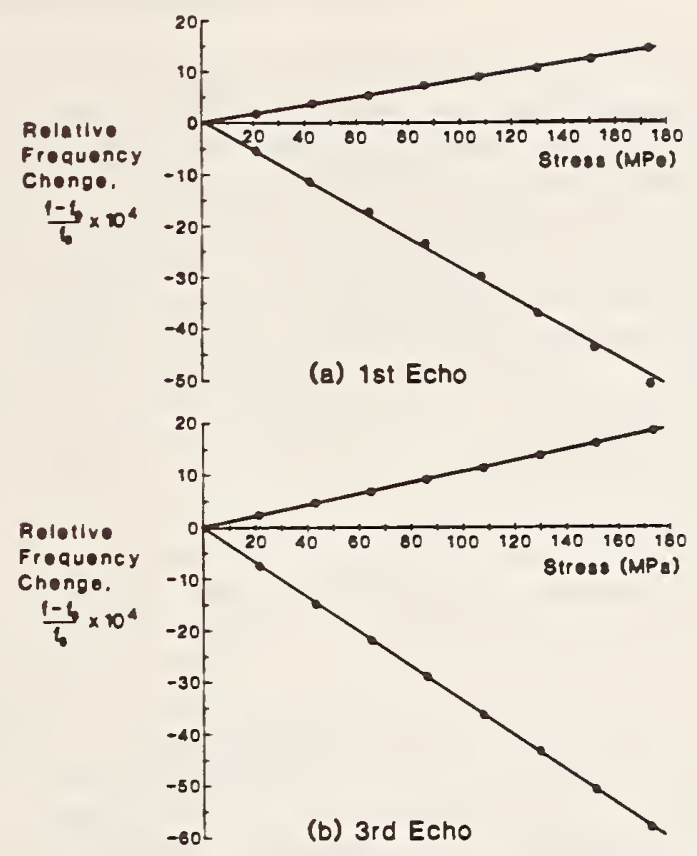

Fig. 6. Typical calibration curves for acoustoelastic constants.

was applied in a servo-hydraulic testing machine. Ultrasonic data were taken with the SH-waves polarized parallel to and perpendicular to the rolling direction. Results for relative frequency change from the unstressed state as a function of applied stress are shown in Fig. 6 for the first and third echoes. The constant a $(\theta)$ is given by the difference of the slopes of the lines from the two orthogonal polarization directions. The calibration values determined were $a\left(33.8^{\circ}\right)=3.7 \times 10^{-5} / \mathrm{MPa}, a\left(12.6^{\circ}\right)=4.4 \times 10^{-5} / \mathrm{MPa}$. The calibration constants were found to vary with position by approximately $1 \%$.

After calibrating the acoustoelastic censtants, velocity measurements were made in the aluminum plate containing a known residual stress state. Measurements of relative velocity change, $\left(\mathrm{SH}_{23}-\mathrm{SH}{ }_{3}\right) / \mathrm{SH}^{\circ}$ as a function of position, were made along a scan ine perpendicular to the rolling direction of the plate and along a radial line from the plug. The difference of the principal stresses, $\left(\sigma_{2}-\sigma_{1}\right)$, was evaluated from the relative velocity measurements and the values of the constants $a\left(33.8^{\circ}\right)$ and $a\left(12.6^{\circ}\right)$ using Eq. (4). This evaluation was possible using this technique despite the fact that an unknown amount of initial anisotropy was present and no measurements were made in the unstressed state. The results for $\left(\sigma_{2}-\sigma_{1}\right)$ are contrasted with the exact theoretical values in $\mathrm{Fig}$. 3 . 
For comparison with the experimental results obtained with the new technique, results obtained using a more conventional application of acoustical birefringence are also plotred in Fig. 5. It was assumed that the anisotropy in the specimen was homogeneous and could be characterized from measurements in an unstressed reference sample. Thus the value of $\left(\mathrm{V}_{\mathrm{S} 2}{ }^{\circ}-\mathrm{V}_{\mathrm{S}}{ }^{\circ}\right) / \mathrm{V}_{\mathrm{s}}{ }^{\circ}$ in $\mathrm{Eq}$. (2) was evaluated on a separate specimen and assumed to be constant. A single relative velocity measurement was then used to infer the value of $\left(\sigma_{2}-\sigma_{1}\right)$. From Fig. 5, it is evident that the assumption of homogeneous anisotropy results in significantly poorer agreement with the exact values of $\left(\sigma_{2}-\sigma_{1}\right)$.

\section{DISCUSSION}

A new experimental procedure has been proposed for using velocity measurements with SH-waves for characterizing residual stress states in anisotropic materials. The theoretical basis for the method has been presented in the form of closed-form expressions for the velocity of SH-waves propagating at arbitrary angles in planes of crystal symmetry. The new procedure is subject to certain limitations, some of which are also present in existing techniques, and some of which are peculiar to this technique:

1. A principal assumption of the theory upon which the proposed procedure is based is that the principal directions of stress are parallel to the crystal axes of the unstressed solid. In many applications of engineering importance this assumption will be valid, for example, the case of butt weldments where the rolling direction is either perpendicular or parallel to the welding direction.

2. Measurements are needed using SH-wave signals propagating at two different angles with respect to the plate normal in order to evaluate $\left(\sigma_{2}-\sigma_{1}\right)$ in the presence of unknown anisotropy. An error propagation analysis was conducted and it was found that the accuracy of the evaluation of $\left(\sigma_{2}-\sigma_{1}\right)$ decreases as the angular difference between the rays corresponding to the two ultrasonic signals decreases. There is thus an interesting trade-off in the proposed technique between precision and spatial resolution. For the measurement precision obtainable using the current experimental apparatus, acceptable precision $(\sim 10 \%)$ in the evaluation of stress can be attained if the angular difference between the two SH-waves rays is $10^{\circ}$ or greater.

3. The error propagation analysis mentioned above also showed that errors in the values of the relative velocity measurements or the acoustoelastic constants would lead to magnified errors in the values of $\left(\sigma_{2}-\sigma_{1}\right)$. For an angle difference of $10^{\circ}$ between the rays used in the measurement it was found that 
$\pm 1 \%$ errors in the measurement would cause approximately $\pm 10 \%$ errors in the value of $\left(\sigma_{2}-\sigma_{1}\right)$. Precision velocity measurement systems are readily capable of making relative velocity measurement to a precision of greater than $1 \%$. However, the use of a separate reference sample to evaluate the acoustoelastic constants can lead to greater than $1 \%$ errors in their values if significant material inhomogeneity is present. If this is found to be a serious problem then it may be necessary to use the in-situ calibration technique proposed by Scott.ll

In conclusion, an experimental procedure has been demonstrated which shows promise for evaluating residual stress states in plate geometries with unknown material property varlations. This is an important step in extending acoustoelastic stress measurements to applications of practical engineering importance.

\section{ACKNOWLEDGMENTS}

The authors are indebted to S. A. Padget, J. Arvidson, Jr., and R. A. Schram, who took much of the experimental data and participated in the construction of the experimental apparatus. The authors are also indebted to J. D. McColskey who prepared the specimens used in the experiments. This work was supported in part by the U.S. Navy, David Taylor Naval Ship Research and Development Center, and the Office of Nondestructive Evaluation of the National Bureau of Standards.

\section{REFERENCES}

1. R.B. King and C.M. Fortunko, "Determination of In-Plane Residual Stress States in Plates Using Horizontally Polarized Shear Waves," submitted to the Journal of Applied Physics.

2. H. Fukuoka, H. Toda and T. Yamone, "Acoustoelastic Stress Analysis of Residual Stress in a Patch-Welded Disk," Exp. Mech. 18, $227,1978$.

3. N.N. Hsu, "Acoustic Birefringence and the Use of Ultrasonic Waves for Experimental Stress Analysis," Exp. Mech. 14, 169, 1974.

4. W.H. Cooper, D.H. Saunderson, C.M. Sayers and M.G. Silk,"Ultrasonics and the Residual Stress Measurement Problem," A.E.R.E. Harwe11, Report No. AERE-R 9588, United Kingdom, 1980.

5. G.S. Kino, J.B. Hunter, G.C. Johnson, A.R. Selfridge, D.M. Barnett, G. Herrmann and C.R. Steele, "Acoustoelastic Imaging of Stress Fields," J. App1. Phys., 50, 2607, 1979.

6. M.P. Scott, D.M. Barnett and D.B. Ilic, "The Nondestructive Determination of Residual Stress in Extruded Billets from Acoustoelastic Measurements," in 1979 Ultrasonics Symp. Proc., J. de Klerk and B.R. McAvoy (eds.), IEEE, New York, p. 265, 1980.

7. O.I. Guscha and F.G. Makhort, "Acoustic Method of Determining Biaxial Residual Stresses," Prikl. Mech. 12, 32, 1976. 
8. A.N. Guz, F.G. Makhort, O.I. Guscha and V.K. Lebedev, "Theory Underlying the Determination of Initial Stresses from the Results of Ultrasonic Measurements," Prikl. Mech. 7, 1205, 1971.

9. M. James and 0 . Buck, "Quantitative Nondestructive Measurements of Residual Stress," CRC Critical Reviews in Solid State Physics and Materials Science 9, 61, 1980.

10. C.M. Kachanov, Foundations of the Theory of Plasticity, MIR Press, Moscow, USSR, P. 127, 1974.

11. M.P. Scott, Doctoral Dissertation, Department of Materials Science and Engineering, Stanford University, Stanford, CA, 1981. 


\title{
Determination of in-plane residual stress states in plates using horizontally polarized shear waves
}

\author{
R. B. King and C. M. Fortunko \\ Fracture and Deformation Division, National Bureau of Standards, Boulder, Colorado 80303
}

(Received 10 September 1982; accepted for publication 18 January 1983)

\begin{abstract}
In this paper a new approach for using acoustic measurements to evaluate residual stresses in the presence of unknown material property variation is presented. Procedures previously applied to the evaluation of stress with acoustic measurements are reviewed, and it is shown that these involve using measurements with bulk waves propagating along the normal to the surface of a plate and do not provide sufficient information to separate the influences of stress and material property variations. To overcome this fundamental limitation, an alternative theory is developed that governs the propagation of shear waves polarized horizontally with respect to the surface of a plate (SH waves), but propagating at oblique angles with respect to the surface normal. The question of separating the effects of residual stress and material properties on acoustic velocity is addressed in detail. A practical experimental procedure is developed that permits the evaluation of the in-plane components of the principal stresses in a plate exhibiting an unknown inhomogeneous initial anisotropy caused by material texture or microstructure. The procedure is then verified experimentally using an aluminum specimen with a known residual stress state, but unknown initial anisotropy.
\end{abstract}

PACS numbers: 46.30.Rc, 43.88.Dv, 43.35.Cg

\section{INTRODUCTION}

In recent years considerable attention has been focused on the possibility of evaluating residual stress states in practical engineering materials by exploiting the fact that the presence of stress influences the velocities of elastic waves in a solid. However, progress has been slow because most practical engineering materials exhibit unknown material property variations, which also influence the elastic wave velocities. In practice, the problem can only be solved by developing a procedure permitting the unambiguous separation of the influences of the main factors determining the elastic wave velocities: metallurgical properties, stress, and temperature.

In this paper the problem of separating the effects of stress and metallurgical variations on the velocities of elastic waves in weakly anisotropic polycrystalline aggregates is addressed. First, using the theory of acoustoelasticity, the effects of stress on elastic waves propagating along the principal stress directions are reviewed. It is shown that restricting measurements to elastic wave propagating along these special directions does not provide sufficient information to separate the effects of stress from those of metallurgical variations. In particular, it is shown that the usual experimental procedure, based on acoustic birefringence, cannot be used to separate the effects of stress and initial anisotropy, caused by microstructure or nonrandom grain orientations in a polycrystalline aggregate (texture). Second, to overcome the above limitation it is proposed that additional information be obtained from measurements of elastic wave velocities along directions that do not coincide with one of the principal stress directions. In particular, it is shown that sufficient information can be obtained using measurements of shear waves propagating in planes of symmetry of the anisotropic, polycrystalline aggregate.
To permit evaluation of stress and anisotropy from the velocity measurements, a special theory of acoustoelasticity is developed. It is shown that the system of equations governing elastic wave propagation in media of orthorhombic symmetry in the presence of stress can be solved numerically. A closed-form theory is also developed that describes the propagation of pure shear waves propagating in the planes of symmetry of the polycrystalline aggregate. Based on this theory, an experimental procedure is developed that permits the evaluation of biaxial stress states in plates when the principal stress directions lie in the planes of symmetry of the anisotropic, polycrystalline aggregate. The proposed experimental procedure has important technological applications. particularly in the nondestructive evaluation of residual stresses in butt weldments.

The proposed experimental procedure is verified using an aluminum plate specimen containing a cylindrical plug that produces a known stress state. A new electromagnetic acoustic transducer (EMAT) configuration is used that permits the generation and detection of horizontally polarized shear waves (SH-waves) from a very wide range of oblique propagation directions, and significantly increases the reproducibility of the measurements. The measured stress distributions are then compared with strain gauge data and calculated values.

\section{THEORY OF ACOUSTOELASTICITY: GENERAL BACKGROUND}

The theory of acoustoelasticity relates measurable changes in the velocities of elastic waves to the stress state in a solid. The theory has been studied by many authors. - A Earlier work was motivated by the need to determine thirdorder elastic constants, which play a significant role in many branches of solid-state physics. More recently, greater atten- 
tion has been focused on the specific application of the theory of acoustoelasticity to the problems of determining stress states in solids. In applying the theory to the determination of residual stress states, it has been commonly assumed that residual stress states are equivalent to applied stress states in terms of their effect on the velocities of elastic waves. This assumption is also used in the present work.

In the past, explicit formulae have been derived that relate the velocities of elastic waves in solids to multiaxial stress states when the principal stress directions and the propagation directions coincide with the crystal axes. ${ }^{3,5,6}$ Most technological applications involve a need to evaluate residual stress states in polycrystalline materials. Microstructure or texture (nonrandom orientation of the grains) result in elastic anisotropy of the polycrystalline aggregates.

For the special case of a plate subjected to a biaxial plane stress state, with the propagation direction along the plate normal, the following relations can be obtained for isotropic solids ${ }^{6}$ :

$$
\begin{aligned}
& \left(v_{1}-v_{l}^{\circ}\right) / v_{1}^{\circ}=A\left(\sigma_{1}+\sigma_{2}\right), \\
& \left(v_{S 2}-v_{S 1}\right) / v_{S}^{\circ}=B\left(\sigma_{2}-\sigma_{1}\right), \\
& \left(v_{S 2}+v_{S 1}-v_{S}^{\circ}\right) / 2 v_{S}^{\circ}=C\left(\sigma_{1}+\sigma_{2}\right),
\end{aligned}
$$

where $v_{l}^{\circ}$ and $v_{S}^{\circ}$ are the longitudinal and shear wave velocities in the absence of stress; the subscript $l$ denotes the longitudinal wave component, and the subscripts $S 2$ and $S 1$ refer to the two shear waves whose polarization directions coincide with the two principal in-plane stress directions $\left(X_{1}\right.$ and $X_{2}$ ). $A, B$, and $C$ are commonly referred to as the acoustoelastic constants, which are functionally related to the secondand third-order elastic constants. The principal stress components are denoted by $\sigma_{1}$ and $\sigma_{2}$.

In anisotropic solids, Eqs. (1)-(3) can be generalized as follows:

$$
\begin{gathered}
\left(v_{1}-v_{l}^{\circ}\right) / v_{1}^{\circ}=A\left(\sigma_{1}+\sigma_{2}\right)+D\left(\sigma_{2}-\sigma_{1}\right) \\
\left(v_{S 2}-v_{S 1}\right) / v_{S}^{\circ}=v_{S 2}^{\circ}-v_{S 1}^{\circ} / v_{S}^{\circ}+B\left(\sigma_{2}-\sigma_{1}\right) \\
\quad+E\left(\sigma_{1}+\sigma_{2}\right) \\
\left(v_{S 2}+v_{S 1}-2 v_{S}^{\circ}\right) / 2 v_{S}^{\circ}=C\left(\sigma_{1}+\sigma_{2}\right)+F\left(\sigma_{2}-\sigma_{1}\right)
\end{gathered}
$$

where $D, E$, and $F$ are additional acoustoelastic constants and $v_{S}^{\circ}$ is the mean shear wave velocity in the absence of stress:

$$
v_{S}^{\circ}=\frac{1}{2}\left(v_{S 2}^{\circ}+v_{S 1}^{\circ}\right)
$$

Equations (4)-(6) are derived in the Appendix for solids exhibiting orthorhombic symmetry. Orthorhombic symmetry has been assumed in the present theory because it is believed that it is sufficiently general to represent most technologically important solids. (It is shown in the Appendix that for weak anisotropies the constants $D, E$, and $F$ are small).

Equation (2) is known as the acoustical birefringence equation and is analogous to the optical birefringence equation used in the theory of photoelasticity. The distinctions between acoustoelasticity and photoelasticity are discussed in detail by Henneke and Green. ${ }^{7}$ The acoustical birefringence effect has been widely used in experimental evaluation of residual stress states. ${ }^{8-10}$ Its main advantages are that only relative measurements of the velocities need be made and that initial specimen inhomogeneities are canceled. However, weak anisotropy can result in large absolute errors in the determination of the quantity $\sigma_{2}-\sigma_{1}$. The errors are caused by the indeterminacy of the $\left(v_{S 2}^{\circ}-v_{S 1}^{\circ}\right) / v_{S}^{\circ}$ term in Eq. (5). The importance of this initial anisotropy effect is widely recognized. Various techniques have been proposed for overcoming this difficulty. ${ }^{1 "}$ To date, none of the above techniques have led to a fully successful experimental demonstration.

As an alternative approach, measurements of the longitudinal wave velocities have been used. ${ }^{12,13}$ An examination of Eq. (4) reveals that the velocity of longitudinal waves is not strongly sensitive ' initial unisotropy. However, initial specimen inhomog itties can cause large errors in the determination of th uantity $\sigma_{2}+\sigma_{1}$. Also, absolute velocity measurements - eeded to determine the absolute magnitude of the quatity $\sigma_{2}+\sigma_{1}$. Scott, Barnett, and $\mathrm{Ilic}^{13}$ have shown that by supplementing the available experimental data by theoretical information, such as the fact that residual stresses must self-equilibrate, the need to obtain absolute velocity data can be eliminated.

Another approach has been proposed by Guscha et $a l .^{6,14}$ for isotropic solids. It was shown theoretically using Eqs. (1)-13) that absolute shear and longitudinal velocity measurements can be used to determine $\sigma_{1}$ and $\sigma_{2}$, separately. In addition, the effects of the initial material inhomogeneity can be eliminated using the above approach. However, in the derivation in Ref. 14, it is assumed that the ratio of $v_{i}^{\circ}$ and $v_{S}^{\circ}$ remains unchanged throughout the specimen. This assumption appears questionable in view of the fact that $v_{S}^{\circ}$ would be more strongly affected than $v_{l}^{\circ}$ by any variations in material anisotropy with position.

In all of the work described above, use is made of bulk elastic waves that propagate along the direction normal to the surface of the plate. This approach is fundamentally limited. In particular, it is not possible to determine uniquely the five unknowns $\sigma_{1}, \sigma_{2}, v_{S 2}^{\circ}, v_{S 1}^{\circ}, v_{l}^{\circ}$ from Eqs. (4)-16).

In the present work, a procedure is proposed that overcomes the above limitations. The procedure is based on making measurements with bulk waves propagating at an angle with respect to the plate normal. In plates, the use of longitudinal (L), and vertically polarized shear (SV) waves in this context would lead to significant difficulties, because such waves are coupled upon reflection and exhibit substantial phase shifts as a function of the angle of incidence. ${ }^{15}$ In addition, an adequate theory for $\mathrm{L}$ and $\mathrm{SV}$ waves in the presence of stress is very complicated. However, the difficulties outlined above can be successfully overcome by using shear waves that are polarized in the plane of the plate (SH waves).

\section{THEORY OF ACOUSTOELASTICITY: MEDIA OF ORTHORHOMBIC SYMMETRY}

The theory of acoustoelasticity for media of orthorhombic symmetry can be developed from the equations of motion for a solid in the presence of stress. In this paper, the approach used in the derivation of the relevant equations of motion follows that of Tokuoka and Iwashimizu. ${ }^{16}$ In this approach, the wave motion is treated as an infinitesimal state 
of deformatton that is superimposed on a large initial, static state of deformation.

Let $X, x$ and $x^{\prime}$ denote the position vectors of a point in an undeformed reference state, the initial deformed state, and the final deformed state, respectively (Fig. 1). The subscripts $i$ and $j$ are defined using the same basis vectors for all three states. In terms of the Cauchy stress, the equations of motion in the final state are given by

$$
\frac{\partial}{\partial x_{j}^{1}}\left(\sigma_{i j}^{1}\right)=\rho^{\prime}\left(\frac{\partial^{2} u_{i}}{\partial t^{2}}+\frac{\partial^{2} u_{i}^{1}}{\partial t^{2}}\right)=\rho^{1} \frac{\partial^{2} u_{i}^{1}}{\partial t^{2}},
$$

where $\rho, \rho^{1}, u_{i}, u_{1}^{1}$, and $\sigma_{i j}, \sigma_{i j}^{1}$ denote density, displacement, and stress, respectively, in the initial and final deformed states. The usual implicit summation convention on repeated indices is used throughout this paper. The nonlinear-elastic constitutive law relating the Cauchy stress to the Lagrangian strain is given by

$$
\sigma_{i j}^{1}=\frac{\rho^{1}}{\rho_{0}} \frac{\partial W^{1}}{\partial E_{r s}^{1}} \frac{\partial x_{i}^{1}}{\partial X_{r}} \frac{\partial x_{j}^{1}}{\partial X_{s}},
$$

where $\rho_{0}$ is the density in the undeformęd state, $E_{r s}^{1}$ the Lagrangian strain, and $W^{\prime}$ the strain energy density in the final state. (The thermodynamic aspects of Eq. (9) are considered in Ref. 3.)

Making use of the strain-displacement relationship

$$
E_{i j}^{1}=\frac{1}{2}\left(\frac{\partial x_{r}^{1}}{\partial X_{i}} \frac{\partial x_{r}^{1}}{\partial X_{j}}-\delta_{i j}\right) \text {, }
$$

and substitutıng for $\sigma_{i j}^{1}$ in Eq. (8), the following expression is obtained:

$$
\left(\bar{C}_{l i k j}+\sigma_{l j} \delta_{i k}\right) \frac{\partial^{2} u_{k}^{1}}{\partial x_{j} \partial x_{l}}=\rho \frac{\partial^{2} u_{i}}{\partial t^{2}} .
$$

In the derivation of the above expression only the lowest order terms in $u_{i}$ and its derivatives have been retained. $\bar{C}_{i j k l}$ is defined by

$$
\bar{C}_{i j k l}=\frac{\rho}{\rho_{0}} \frac{\partial^{2} W}{\partial E_{r s} \partial E_{p q}} \frac{\partial x_{i}}{\partial X_{r}} \frac{\partial x_{j}}{\partial X_{s}} \cdot \frac{\partial x_{k}}{\partial X_{p}} \frac{\partial x_{l}}{\partial X_{q}} .
$$

(In the derivation of Eq. (11) it has been assumed that $\bar{C}_{i j k l}$ and $\sigma_{i j}$ are homogeneous.)

Next, a plane wave solution is assumed for $u_{i}^{1}$ :

$$
u_{i}^{1}=d_{i} e^{i \beta}\left(x_{l} p_{l}-v t\right)
$$

where $\beta$ is the wave number, $p_{i}$ the direction cosine of the wave normal, and $d_{i}$ the unit vector in the direction of particle motion. A substitution of Eq. (13) into Eq. (11) leads to the generalized Christoffel's equations:

$$
\left[\bar{C}_{i j k l} p_{i} p_{l}+\left(\sigma_{i l} p_{i} p_{l}-\rho v^{2}\right) \delta_{j k}\right] d_{k}=0 .
$$

The eigenvalues of Eq. (14) are

$$
\rho v^{2}-\sigma_{i l} \rho_{i} \rho_{l},
$$

and the eigenvectors are the particle displacement vectors $d_{k}$. It is tempting to rewrite Eq. (14) in the form

$$
\left[\left(\bar{C}_{i j k l}+\sigma_{i l} \delta_{j k}\right) p_{i} p_{l}-\rho v^{2}\right] d_{i}=0,
$$

and define the effective stiffness tensor

$$
C_{i j k l}^{\mathrm{eff}}=\bar{C}_{i j k l}+{ }^{\prime} \sigma_{i l} \delta_{j k}
$$

However, this interpretation is misleading, because the effective stiffness tensor does not exhibit the familiar symmetry properties. ${ }^{4}$ For example, in general

$$
C_{1212}^{\mathrm{eff}_{12}} \neq C_{2121}^{\mathrm{eff}} \text {. }
$$

Thus, in the presence of stress, a shear wave propagating along the $x_{1}$ direction and polarized along the $x_{2}$ direction will not have the same velocity as a shear wave propagating in the $x_{2}$ direction and polarized along the $x_{1}$ direction. Therefore, only Eq. (14) will be used in the subsequent discussion.

The strain energy density $W$ can be expressed as Eq. (17):

$$
W=\frac{1}{2} C_{i j k l} E_{i j} E_{k l}+\frac{1}{6} C_{i j k l m n} E_{i j} E_{k l} E_{m n},
$$

where $C_{i j k / m n}$ is the third-order stiffness tensor. Substituting into Eq. (12), the following expression for $\bar{C}_{i j k l}$ is obtained:

$$
\begin{aligned}
\vec{C}_{i j k l}= & \frac{\rho}{\rho_{0}}\left(C_{m n p q}+C_{m n p q r s} E_{r s}\right) \\
& \times \frac{\partial x_{i}}{\partial X_{m}} \frac{\partial x_{j}}{\partial X_{n}} \frac{\partial x_{k}}{\partial X_{p}} \frac{\partial x_{l}}{\partial X_{q}} .
\end{aligned}
$$

Equation (18) can be linearized at this point. Thus

$$
E_{i j} \approx S_{i j k l} \sigma_{k l} \text {, }
$$

where $S_{i j k l}$ is the elastic compliance tensor, and to first order in $\partial \mu_{i} / \partial X_{j}$

$$
\frac{\partial x_{i}}{\partial X_{j}} \approx E_{i j}+\Omega_{i j}+\delta_{i j},
$$

where $\Omega_{i j}$ is the local rigid body rotation.

In general, for propagation directions that do not lie in the planes of symmetry, the eigenvalue problem of Eq. (14) cannot be solved in closed form. However, numerical solutions can be obtained quite easily. Once the stress tensor $\sigma_{t}$ is specified, the tensor $\bar{C}_{i j k l}$ can be calculated. Then, for each propagation direction $p_{i}$, the product $\bar{C}_{i j k l} p_{1} p_{l}$ can be obtained, resulting in a real three by three matrix whose eigenvalues and eigenvectors can be found. Computational subprocedures for solving this type of eigenvalue problem are readily available. ${ }^{18}$

Closed-form solutions of the eigenvalue problem can be obtained when the propagation direction is parallel to the material symmetry axes and the $x_{1}, x_{2}$ and $x_{3}$ axes are parallel to the principal stress directions. The solutions are derived in the Appendix and the results are given by Eqs. (4)(6).

Closed-form solutions of the eigenvalue problen are also obtainable when the propagation direction lies in a plane of material symmetry. This is a consequence of the fact that in the planes of material symmetry, the wave whose particle displacements are normal to the plane of matcrial symmetry is a pure mode. Planes of material symmetry in the absence of stress will remain planes of material symmctry in the presence of stress when the principal stress direc. tions are parallel to the material symmetry axes.

Consider a plane wave propagating in the $x_{2}-x_{3}$ plane in a medium of orthorhombic symmetry $w$ here $x_{1}, x_{2}$ and $x_{1}$ are the principal stress directions. In this case the eigenvalue 
problem Eq. (14) simplifies to

$$
\begin{aligned}
& \left\{\left(\bar{C}_{2 j k 2} p_{2}^{2}+2 \bar{C}_{2 j k 3} p_{2} p_{3}+\bar{C}_{3 j k 3} p_{3}^{2}\right)\right. \\
& \left.\quad+\left[\sigma_{22} p_{2}^{2}+\sigma_{33} p_{3}^{2}-\rho v^{2}\right]\right\} d_{k}=0 .
\end{aligned}
$$

The elements of the matrix enclosed by the parentheses vanish when $K=2, j \neq 1$, or $j=1, k \neq 1$. This can be verified by using Eq. (18) and examining the symmetry of the tensors $C_{i j k l}$ and $C_{i j k l m n}$. The symmetry properties of the tensor $C_{i j k l m n}$ are given in Ref. 19. The SH wave, which is denoted by $d_{1}=1, d_{2}=0, d_{3}=0$, is then a pure mode that can easily be split off and treated separately. The velocity of this mode is given by

$\rho v^{2}=\bar{C}_{2112} p_{2}^{2}+2 \bar{C}_{2113} p_{2} p_{3}+\bar{C}_{3113} p_{3}^{2}+\sigma_{22} p_{2}^{2}+\sigma_{33} p_{3}^{2}$,

where $\bar{C}_{2113}$ vanishes as a result of the assumed symmetry.

Next, making use of Eq. (18) and noting that in the principal coordinates $x_{1}, x_{2}$, and $x_{3}$

$$
\frac{\partial x_{i}}{\partial X_{k}}=\delta_{i k}\left(1+E_{i i}\right) \text { (no sum on } i \text { ), }
$$

it can be shown that

$$
\begin{aligned}
\mathrm{SH}_{23}^{2}= & \frac{\sin ^{2} \phi}{\rho_{0}}\left[C_{66}\left(1+2 E_{11}+2 E_{22}\right)\right. \\
& \left.+C_{661}\left(E_{11}+E_{22}\right)\right] \\
& \left.+C_{663} E_{33}+\sigma_{22}\right]+\frac{\cos ^{2} \phi}{\rho_{0}} \\
& \times\left[C_{55}\left(1+2 E_{11}+2 E_{33}\right)\right. \\
& \left.+C_{661}\left(E_{11}+E_{33}\right)+C_{441} E_{22}+\sigma_{33}\right],
\end{aligned}
$$

where $\phi$ is defined as the angle between the propagation direction and the $x_{3}$ axis, and the contracted (Voigt's) notation is used. For further convenience, a new notation is introduced: $\mathrm{SH}_{i j}$ is taken to denote the velocity of the $\mathrm{SH}$ wave propagating in the $x_{i}-x_{j}$ plane.

The eigenvalue problem then reduces to a system of two equations for $j, k=2,3$. Upon expanding, a quadratic is obtained for the eigenvalue $\left(\rho v^{2}-\sigma_{1} p_{2}^{2}-\sigma_{33} p_{3}^{2}\right)$, which can be solved for the velocity in closed form. However, obtaining a solution is tedious and not required for subsequent development.

In a manner analogous to that used in deriving Eq. (24), it can be shown that

$$
\begin{aligned}
\mathrm{SH}_{13}^{2}= & \frac{\sin ^{2} \phi}{\rho_{0}}\left[C_{66}\left(1+2 E_{11}+2 E_{22}\right)\right. \\
& \left.+C_{661}\left(E_{11}+E_{22}\right)+C_{663} E_{33}+\sigma_{11}\right] \\
& +\frac{\cos ^{2} \phi}{\rho_{0}}\left[C_{44}\left(1+2 E_{22}+2 E_{33}\right)+C_{441} E_{11}\right. \\
& \left.+C_{661}\left(E_{22}+E_{33}\right)+\sigma_{33}\right] .
\end{aligned}
$$

For experimental purposes, it is convenient to express Eqs. (24) and (25) in terms of differences in the velocities. Only terms to first order in the difference between the velocities in the absence and presence of stress are retained. Velocities in the unstressed state are denoted by the superscript 0 . The full derivation is given in the Appendix and only the results are repeated below:

$$
\begin{aligned}
& \left.\frac{\mathrm{SH}_{23}-\mathrm{SH}_{13}}{\mathrm{SH}^{0}}\right|_{\phi} \\
& =\frac{\cos ^{2} \phi}{2 \rho\left(\mathrm{SH}^{0}\right)^{2}}\left(C_{55}-C_{44}\right)+a(\phi)\left(\sigma_{2}-\sigma_{1}\right) \\
& \quad+c(\phi)\left(\sigma_{2}+\sigma_{1}\right),
\end{aligned}
$$

and

$$
\begin{aligned}
& \left.\frac{\mathrm{SH}_{23}+\mathrm{SH}_{13}-2 \mathrm{SH}^{\mathrm{o}}}{2 \mathrm{SH}^{0}}\right|_{\phi}=b(\phi)\left(\sigma_{2}+\sigma_{1}\right) \\
& +d(\phi)\left(\sigma_{2}-\sigma_{1}\right) .
\end{aligned}
$$

For weak anisotropies, $c$ and $d$ are the first order in $\epsilon$, where $\epsilon$ is a parameter characterizing the anisotropy [e.g., $\left.C_{55}=C_{44}(1+\epsilon)\right]$. Thus, $c$ and $d$ can be neglected.

In the above discussion $\mathrm{SH}^{\circ}$ denotes the mean unstressed velocity, which is explicitly given by

$$
\begin{aligned}
\mathrm{SH}^{0}=\frac{1}{2} & {\left[\left(\frac{C_{44} \cos ^{2} \phi+C_{66} \sin ^{2} \phi}{\rho_{0}}\right)^{1 / 2},\right.} \\
& \left.+\left(\frac{C_{55} \cos ^{2} \phi+C_{66} \sin ^{2} \phi}{\rho_{0}}\right)^{1 / 2}\right] .
\end{aligned}
$$

An estimate of $\mathrm{SH}^{0}$ may be used in the denominator of Eqs. (26) and (27), because a small error in the denominator results in a higher order error in the calculated stress states. However, the exact value of $\mathrm{SH}^{0}$ is needed in the numerator of Eq. (27) because a small error in the numerator results in a large error in the calculated stress states.

Equation (26) is a generalization of the acoustical birefringence relation [Eq. (5)] and, in fact, reduces to it for the special case $\phi=0$. Similarly, Eq. (27) is a generalization of Eq. (6). The importance of Eqs. (26) and (27) is that the same unknowns are present regardless of the value of $\phi$. As a consequence, measurements at different values of $\phi$ provide additional information without increasing the number of unknowns. Equation (26) is particularly important because only two unknowns are present: $\left(C_{55}-C_{44}\right)$ and $\left(\sigma_{2}-\sigma_{1}\right)$. Thus, measurements at only two values of $\phi$ are required to evaluate the quantity $\left(\sigma_{2}-\sigma_{1}\right)$.

\section{EXPERIMENTAL PROCEDURE}

It follows from the preceding discussion that the residual stress state and the relevant elastic constants can be uniquely determined by making precise velocity measurements with off-axis SH waves in the planes of material symmetry. To separate the effects of texture and stress, measurements must be made in mutually orthogonal material symmetry planes.

It is straightforward to determine the difference between two principal stresses by making relative measurements and using Eq. (26). In this case, the procedure involves first making a velocity measurement of an $\mathrm{SH}$ wave propagating in the $x_{2}-x_{3}$ plane at an angle $\phi$ with respect to $x_{3}$ axis. Next, the measurement is repeated in the $x_{1}-x_{3}$ plane for the same angle $\phi$. Both measurements must then be repeated for a different value of $\phi$. The difference between the two measurements is then used in Eq. (26) to evaluate $\left(\sigma_{2}-\sigma_{1}\right)$ and the quantity $\left(C_{55}-C_{44}\right)$. (In many technological applications the knowledge of $\left(\sigma_{2}-\sigma_{1}\right)$ is sufficient because addi- 
thomal information related to the specimen geometry is usual1) avallable to estimate the relative magnitudes of $\sigma_{2}$ and $\sigma_{1}$ ).

In principle, the separate values of $\sigma_{2}$ and $\sigma_{1}$ can be deternmed experimentally by using Eqs. (26) and (27). However, in this case a set of five independent measurements must be performed at different values of $\phi$. The five measurements are needed to determine the values of $\sigma_{1}, \sigma_{2}, C_{44}, C_{55}$, and $C_{\infty}$. In practice, an additional measurement may be required to compensate for any path length variations.

In practical applications, small errors $\epsilon_{1}, \epsilon_{2}$ can be present in the values of $\mathrm{SH}_{23}-\mathrm{SH}_{13} / \mathrm{SH}^{0}$ measured at angles $\phi_{1}$ and $\phi_{2}$, respectively, and errors $\epsilon_{3}, \epsilon_{4}$ can be present in $a\left(\phi_{1}\right)$ and $\left.a \mid \phi_{2}\right)$ in Eq. (26). Inverting the two equations obtained from the measurements at $\phi_{1}$ and $\phi_{2}$ to evaluate $\sigma_{2}-\sigma_{1}$ results in

$$
\begin{aligned}
\left(\sigma_{2}-\sigma_{1}\right) & \\
= & \left(\sigma_{2}-\sigma_{1}\right)^{\mathrm{ex}}\left[1+\left(\frac{\delta_{1} \epsilon_{1} C_{2}-\sigma_{2} \epsilon_{2} C_{1}}{\delta_{1} C_{2}-\delta_{2} C_{1}}\right.\right. \\
& \left.\left.+\frac{\epsilon_{4} a_{2} C_{1}-\epsilon_{3} a_{1} C_{2}}{a_{1} C_{2}-a_{2} C_{1}}\right)\right],
\end{aligned}
$$

where $\left(\sigma_{2}-\sigma_{1}\right)^{\text {ex }}$ is the exact value of $\sigma_{2}-\sigma_{1} ; \delta_{1}, \delta_{2}$ are the values of $\left(\mathrm{SH}_{23}-\mathrm{SH}_{13}\right) / \mathrm{SH}^{0}$ at $\phi_{1}$ and $\phi_{2}, a_{i}=a\left(\phi_{i}\right)$, and $C_{t}=\cos ^{2}\left(\phi_{i}\right)$. The term in the parentheses represents the relative error in stress caused by the accumulation of errors in velocity measurements and calibrations. The most severe case of error magnification occurs when $\epsilon_{1}=-\epsilon_{2}$ and $\epsilon_{3}=\epsilon_{4}$. In this case, Eq. (29) becomes

$$
\begin{aligned}
\left(\sigma_{2}-\right. & \left.\sigma_{1}\right) \\
& =\left(\sigma_{2}-\sigma_{1}\right)^{\mathrm{ex}}\left\{1+\left[\frac{\delta_{1} C_{2}+\delta_{2} C_{1}}{\delta_{1} C_{2}-\delta_{2} C_{1}} \epsilon_{1}\right.\right. \\
& \left.\left.+\left(\frac{a_{2} C_{1}+a_{1} C_{2}}{a_{1} C_{2}-a_{2} C_{1}}\right) \epsilon_{4}\right)\right\} .
\end{aligned}
$$

To obtain realistic numbers for insertion in Eq. (30), anisotropic second-order elastic constant values for steel and aluminum were obtained by slightly perturbing nominal isotropic values. The third-order elastic tensor was taken to be isotropic, and the third-order constants $l, m$, and $n$ were taken from Ref. 24. The acoustoelastic constants $a(\phi)$ were calculated from the second-and third-order elastic constants using the expression in the Appendix. Using the values for aluminum, for $\phi_{1}=10^{\circ}$ and $\phi_{2}=15^{\circ}$, the error term then becomes $-61 \epsilon_{1}-71 \epsilon_{2}$. It follows that errors in the relative velocity measurements or in the calibration of the constants $a(\phi)$ cause errors of two orders of magnitude larger in $\sigma_{2}-\sigma_{1}$. If $\phi_{1}=10^{\circ}$ and $\phi_{2}=20^{\circ}$, the error term is $-9 \epsilon_{1}-11 \epsilon_{2}$. Thus, the error magnification is greatly reduced as the angular difference between two successive measurements is increased. (Similar results were obtained using the elastic constants for steel.)

Measurement imprecision of less than $1 \%$ can be obtained with the relative velocity measurement system used in the present work. From the above, it follows that $\left(\sigma_{2}-\sigma_{1}\right)$ can be determined with an error of less than $10 \%$. To apply Eqs. (26) and (27), the functional forms of $a(\phi)$ and $b(\phi)$ must be known in advance. As shown in the Appendix, the knowledge of $a(\phi)$ and $b(\phi)$ at $\phi=0^{\circ}$ and $90^{\circ}$ is all that is required to determine the unique functional forms for $a(\phi)$ and $b(\phi)$. Unfortunately, published values of third-order elastic constants to evaluate $a(\phi)$ and $b(\phi)$ at $\phi=0^{\circ}$ and $90^{\circ}$ are not applicable because nominally similar engineering alloy's can exhibit large variations of the acoustoelastic constants. ${ }^{10.20} \mathrm{As}$ a consequence, the values of the acoustoelastic constants must be calibrated directly on the material under study.

In principle, the required calibration can be performed on a separate reference sample subjected to known applied stresses, but nominally of the same composition as the specimen whose residual stress state is to be determined. However, slight variations in the values of the acoustoelastic constants may still be present in the specimen to be examined. This uncertainty would normally be expected to result in errors of the same order in the calculated values of $\sigma_{1}$ and $\sigma_{2}$. However, as discussed below, magnification of error can occur if measurements at different angles are used to evaluate stresses. If errors are expected to be of such a magnitude that they cannot be tolerated, then an in situ calibration technique of Scott can be used. ${ }^{22}$

\section{PRACTICAL EXPERIMENTAL CONFIGURATION}

To obtain experimental data for utilizing Eqs. (26) and (27), transducers of SH waves are required that produce ultrasonic beam patterns with wide angular distributions. Recent technological advances have made it feasible to construct such transducers. A practical transducer design is shown in Fig. 1.

The transducer configuration as shown in Fig. 1 is derived from the periodic permanent magnet EMAT that has been developed for nondestructive evaluation (NDE) applications. ${ }^{23.24}$ However, to produce a compact impulse response and ultrasonic beams with a wide angular pattern, the transducer of Fig. 1 employs only one $(M=1)$ pair of permanent magnets. The principles of operation and design details are described in detail elsewhere. ${ }^{24,25}$ Instead, in this paper. attention is focused on the ability of the new EMAT configuration to produce wide angular beam patterns and. by reciprocity, to receive ultrasonic signals from a wide range of angles.

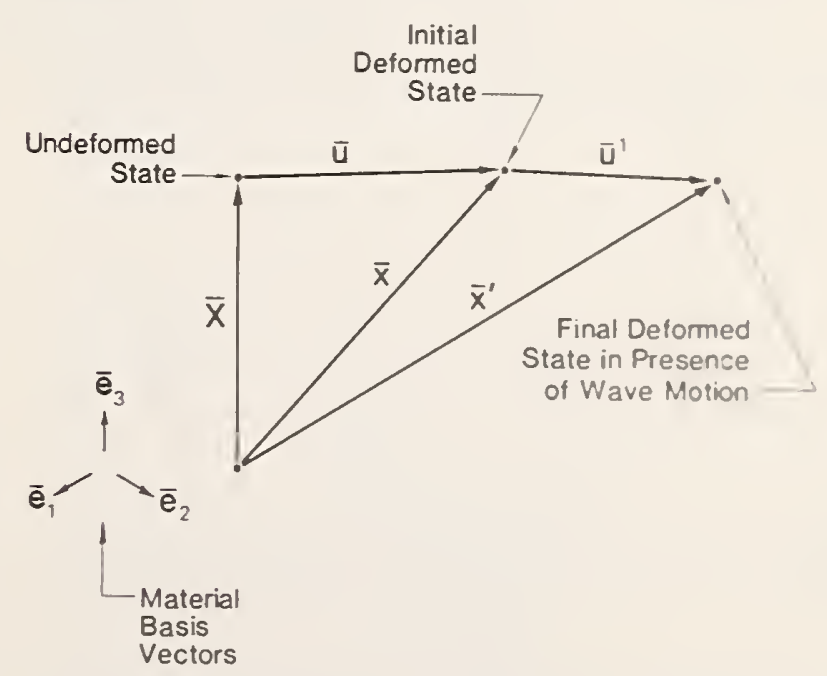

FIG. 1. Geometry used for acoustoelasicisy derisatıon 


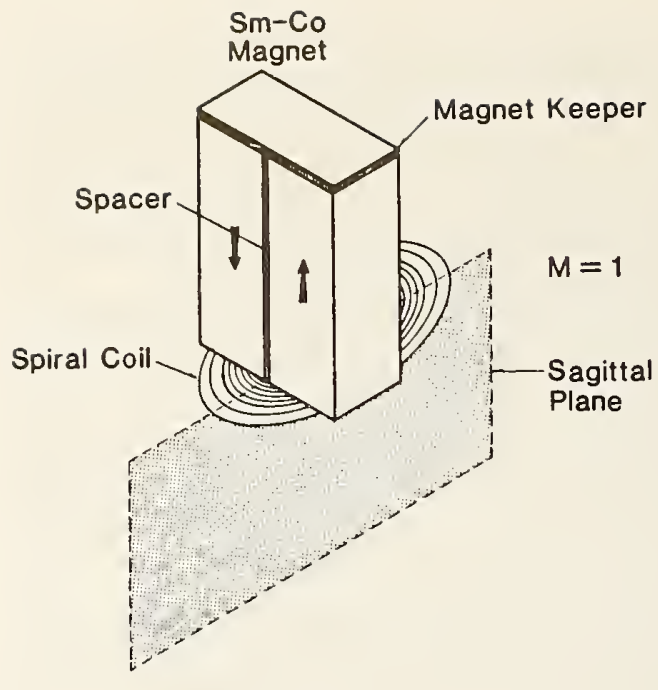

FIG. 2. Practical electromagnetic-acoustic transducer design.

A representative ultrasonic radiation pattern of the EMAT depicted in Fig. 1 is shown in Fig. 2. In Fig. 2, the transmitted ultrasonic amplitude (at $520 \mathrm{kHz}$ ) is plotted as a function of the angles $(\phi)$ in the plane normal to the surface of a plate and bisecting the transducer between the magnets (the sagittal plane). It is evident that the radiation pattern is very broad and, in particular, does not vanish at any angle in the sagittal plane. By reciprocity, the same directivity pattern applies upon reception. As a consequence, in the sagittal plane, the simple EMAT of Fig. 1 acts in a manner similar to that of a finite line-source of $\mathrm{SH}$ waves that is positioned on the surface of the plate. This property of the transducer is needed to study the velocities of ultrasonic waves as a function of propagation direction for two reasons. First, it permits the collection of ultrasonic velocity data from a wide range of angles. Second, it reduces the influence of diffraction effects, which must often be taken into account in accurate velocity measurements. ${ }^{25-27}$ (The diffraction properties of elementary and practical SH-wave EMATs are discussed in detail by Pardee and Thompson. ${ }^{28}$ )

A practical experimental configuration for determining stress states in anisotropic plates is shown in Fig. 3. In the configuration of Fig. 3 two SH-wave EMATs are used. One

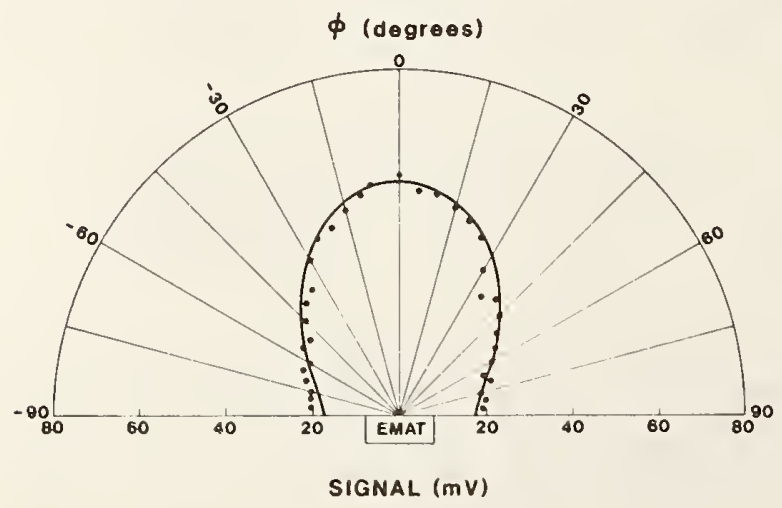

FIG. 3. Ultrasonic radiation pattern of electromagnetic-acoustic transducer. of the EMATs is used as a generator and the other as a receiver of the probing ultrasonic signals. Because the two EMATs are aligned along the same sagittal plane, the receiver EMAT is principally sensitive to ultrasonic signals with a displacement component parallel to the surfaces of the plate and normal to the sagittal plane (SH waves).

In the special case when one of the principal stress directions is parallel to a crystal axis and the sagittal plane is also a plane of crystal symmetry, the SH-wave signals propagate are pure modes. Since $\mathrm{SH}$-waves are decoupled from longitudinal $(\mathrm{L})$ and vertically polarized shear $(\mathrm{SV})$ waves upon reflection, they are not phase shifted upon reflection from the surfaces of the plate. This important observation results in a significant simplification in the inversion of the experimental data and forms the basis for the experimental determination of residual stress states in planar geometries.

An examination of Fig. 3 reveals that many independent ultransonic signal paths (rays) exist that connect the centers of the generator and receiver EMATs. In fact, useful experimental information is available over a range of angles $\phi$, from grazing $\left(\phi=90^{\circ}\right)$ to nearly normal $\left(\phi \sim 0^{\circ}\right)$. As a consequence, by measuring the phases of a number of the ultrasonic signals in planes of symmetry, sufficient information is provided to permit the unambiguous determination of the in-plane stress components, elastic constants and adjustment for plate thickness variations, if needed.

\section{EXPERIMENTAL VERIFICATION}

To verify the experimental procedure, measurements were carried out on an aluminum plate specimen, $25 \mathrm{~mm}$ thick, which contained a known biaxial stress state and unknown initial anisotropy. Aluminum was chosen for this study because it is typical of engineering materials that although nominally elastically isotropic, exhibit a small amount of anisotropy. The anisotropy causes an effect on velocity that is not negligible in comparison to the effect of stress. The design of the experimental specimen is shown in Fig. 5. The test plate was square, $686 \mathrm{~mm}$ on each side, and made of type 6061-T6 aluminum. In the center of the plate is a hole into which was inserted a circular plug, $102 \mathrm{~mm}$ diameter, made of type 304 stainless steel. Prior to insertion, the stainless steel plug was cooled to $77 \mathrm{~K}$ and the aluminum test plate heated to $423 \mathrm{~K}$. After insertion, the resulting strains were monitored with strain gauges placed along radial lines until the whole test specimen was returned to the ambient temperature. The strain gauge readings confirmed the theoretically predicted stress distribution.

To obtain ultrasonic data, the specimen shown in Fig. 5 was scanned using the experimental SH-wave EMAT configuration, as shown in Fig. 4. The scanning was carried out along one of the radial lines that coincide with the axis of plate symmetry, as shown in Fig. 5. To measure the phases of the ultrasonic signals, a modified system of Kino et al. ${ }^{11}$ was used. In this system the relative frequency change required to maintain a constant phase difference between an acoustic signal and a reference signal is measured. Relative velocity changes can then be evaluated from the relation 


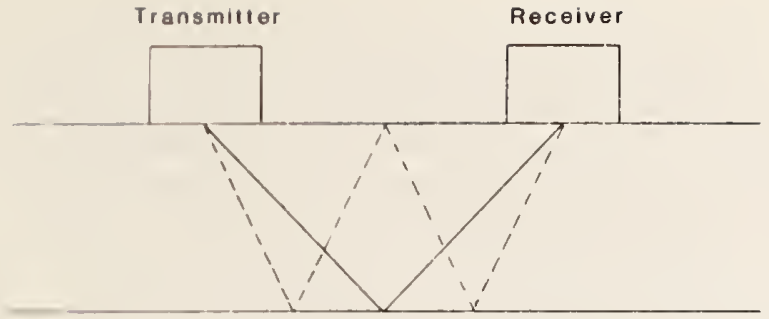

FIG. 4. Transducer configuralion for siress measuremenl.

$$
\frac{\Delta V}{V}=\frac{\Delta f}{f}+\frac{\Delta t}{t},
$$

where $f$ denotes frequency and $t$ plate thickness. Measurements were made using $850-\mathrm{kHz} \mathrm{SH}$-wave probing signals with a $10 \%$ fractional bandwidth. It should be added that the scanning was performed manually, using a grid laid out on the surface of the test specimen without the aid of mechanical positioning mechanisms.

The relative velocity differences $\left(\mathrm{SH}_{23}-\mathrm{SH}_{13}\right) / \mathrm{SH}^{0}$ were calculated for several positions along the scan line, with the $x_{2}$ axis taken to coincide with the plate rolling direction. The ultrasonic data were obtained from the first and third arrival signals, as shown in Fig. 4, with the SH-wave EMAT pair aimed along the scan line, and, then, at $90^{\circ}$ with respect to the scan line. The first and third arrivals were selected because of the large difference in the angle defined by their rays and the plate surface normal, $33.8^{\circ}$ and $12.6^{\circ}$. The difference in the principal stresses, $\sigma_{2}-\sigma_{1}$, was then evaluated from the velocity measurements using Eq. (26). As discussed in the preceding section, the constant $c(\phi)$ was neglected because of the weak anisotropy. The values of the constants $a\left(\phi_{1}\right)$ and $a\left(\phi_{2}\right)$ were determined independently using tensile calibration specimens obtained from the ends of the test block shown in Fig. 5. Tension was applied parallel to the rolling direction in a servohydraulic testing machine. Measurements of relative frequency change as a function of applied stress for an SH-wave polarized parallel and perpendicular to the rolling direction were made. The results are plotted in Fig. 6. The values of the constant $a(\phi)$ is given by
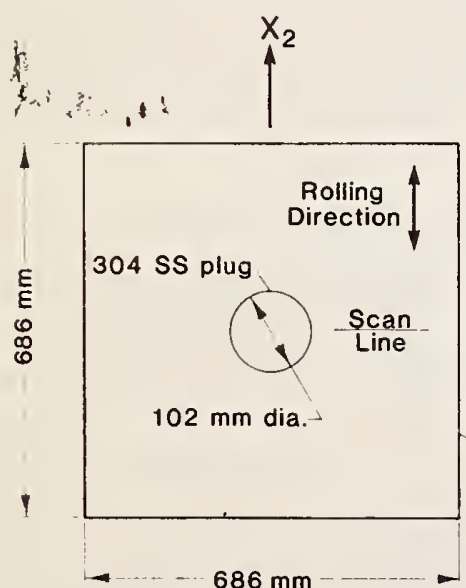

Aluminum 6061 Plate $25 \mathrm{~mm}$ thick

FIG. 5. Specimen containing a known residual stress state.
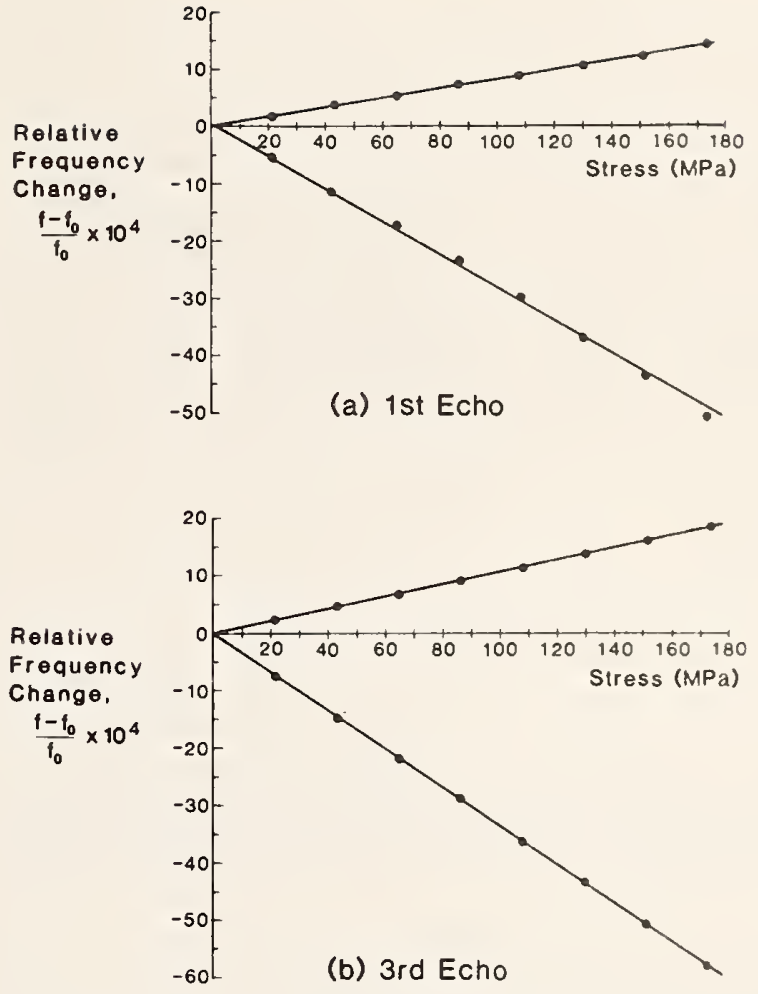

FIG. 6. Typical calibration curves for acoustoelastic constants.

the differences of the slopes of the line plotted. Measurements were taken using the first and third echoes from the back surface of the specimen. The calibration values determined were $a\left(33.8^{\circ}\right)=3.7 \times 10^{-5} \quad \mathrm{MPa}$, $a\left(12.6^{\circ}\right)=4.4 \times 10^{-5} \mathrm{MPa}$. The calibration constants were found to vary with position, but the variations were less than $1 \%$. Therefore, they were judged acceptable and in situ calibration was not needed. The experimental results for the ultrasonically measured values of $\sigma_{2}-\sigma_{1}$ are shown in Fig. 7. For comparison, the calculated values of $\left(\sigma_{2}-\sigma_{1}\right)$ from the elasticity solution are also shown in Fig. 7. The calculated distribution is parabolic, and the experimental results are seen to follow this shape.

As an additional comparison, the experimental results obtained with obliquely propagating $\mathrm{SH}$ waves were related to results that would have been obtained using one of the conventional procedures, based on acoustical birefringence which are also plotted in Fig. 7. It was assumed that the texture was homogeneous and could be characterized using measurements on unstressed reference specimens. Correspondingly, the value of $\left(V_{S 2}{ }^{\circ}-V_{S 1}{ }^{\circ}\right) / V_{S}{ }^{\circ}$ in Eq. (5) was obtained using an unstressed specimen. Then, the values of $\left(V_{S 2}-V_{S 1}\right) / V_{S}{ }^{\circ}$ were measured on the test specinen showil in Fig. 4. It is clear that the assumption of homogeneous texture results in poor agreement between the ultrasonic measurements and the calculated stress statc. Tlis result was expected because the texture was measured ultrasome.s! ly in an unstressed plate of specinen materlal and found io be inhomogeneous. The values obtaned usug llec contentional procedure were lower than the values obtanticel usmg theory and strain gauges. Therefore, it is beleved that the 


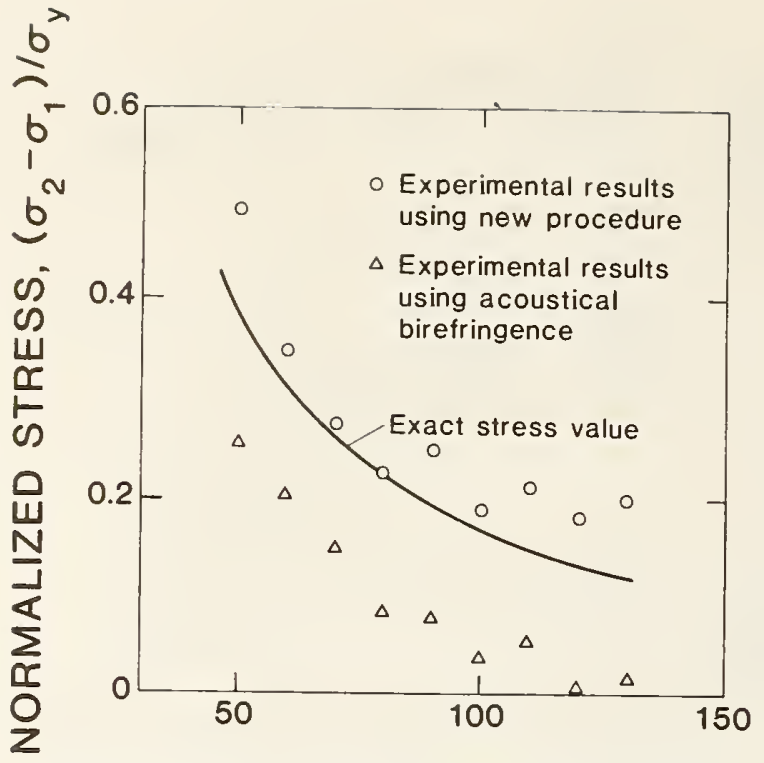

DISTANCE FROM PLUG'BOUNDARY,mm

FIG. 7. Principal difference evaluated in specimen of Fig. 5.

new experimental procedure would be inherently more accurate than the conventional procedure in practical engineering applications. In many such applications (e.g., welding and forming) significant variations of texture with position are expected.

\section{DISCUSSION}

Closed-form expressions relating the velocities of elastic waves propagating at arbitrary angles in the planes of symmetry of weakly anisotropic, polycrystalline solids have been presented. In particular, it has been shown that using measurements of the velocities of pure shear waves as a function of propagation direction, sufficient information is obtained to permit the evaluation of residual stress states in a practical plate geometry. In fact, measurements at only two independent propagation directions are required to obtain the difference in the two in-plane principal stress components. Furthermore, an experimental procedure, based on the new theory, has been proposed and evaluated using a new SH-wave EMAT design. However, the new experimental procedure is subject to certain limitations.

(1) One of the principal assumptions of the new theory is that the principal stress directions are parallel to the crystal axes of the unstressed anisotropic solid. If this condition is violated the $\mathrm{SH}$ waves in a plate are no longer pure shear waves and the theory cannot be assumed to be correct. However, in many applications of technological importance the assumption of coincidence of the symmetry planes with the principal stress directions is valid. Prominent examples involve most butt weldments in which the rolling direction is either perpendicular or parallel to the welding direction. In addition, this assumption is also present in the commonly used acoustical birefringence method, and thus does not limit the proposed procedure more than conventional procedures.
(2) Measurements using $\mathrm{SH}$-wave signals propagating at two different angles with respect to the plate normal are required to obtain the difference of the two principal stresses $\sigma_{1}$ and $\sigma_{2}$ in the presence of unknown anisotropy. The accuracy of such measurements increases in proportion to the angular difference between the rays corresponding to the two ultrasonic signals, because the matrix relating $\left(\sigma_{2}-\sigma_{1}\right)$ and anisotropic effects becomes singular for $\Delta \phi=0$. However, it has been shown that an acceptable accuracy $(\sim 10 \%)$ can be attained if the angular difference between the two SHwave rays is $20^{\circ}$ or greater. As a consequence, the spatial resolution of the new experimental system is directly determined by the thickness of the test specimen.

In conclusion, an experimental procedure has been demonstrated that can be used to determine the residual stress states in plate geometries. This is an important step in extending the theory of acoustoelasticity to practical applications of major technological importance.

\section{ACKNOWLEDGMENTS}

The authors are indebted to S. A. Padget, J. Arvidson, $\mathrm{Jr}$., and R. A. Schramm, who participated in the construction of the experimental apparatus and in the experiments described. The authors are also indebted to J. D. McColskey who prepared the specimens used in the experiments. This work was supported in part by the U.S. Navy, David Taylor Naval Ship Research and Development Center, and the Office of Nondestructive Evaluation of the National Bureau of Standards.

\section{APPENDIX: DERIVATION OF ACOUSTOELASTIC RELATIONS FOR A MEDIUM OF ORTHORHOMBIC SYMMETRY}

Explicit formulae for velocities of waves propagating in specific directions are obtained by substituting for $p_{i}$ in Eq. (14) and making use of Eq. (18). For the case of a wave propagating parallel to the $x_{3}$ crystal axis, Eq. (14) reduces to

$$
\left[C_{3 j k 3}+\left(\sigma_{33}-\rho v^{2}\right) \delta_{j k}\right] d_{k}=0
$$

Furthermore, by considering the symmetry of the tensors $C_{i j k l}$ and $C_{i j k l m n}$ it can be shown that the elements of the matrix in brackets vanish for $j \neq k .^{24}$ Thus, there are three uncoupled modes whose velocities are given by

$$
\begin{aligned}
V_{l}^{2}= & \frac{1}{\rho_{0}}\left[C_{33}\left(1+4 E_{33}\right)+\sigma_{33}+C_{331} E_{11}\right. \\
& \left.+C_{332} E_{22}+C_{333} E_{33}\right], \\
V_{S 2}^{2}= & \frac{1}{\rho_{0}}\left[C_{44}\left(1+2 E_{33}+2 E_{22}\right)+\sigma_{33}+C_{441} E_{11}\right. \\
& \left.+C_{442} E_{22}+C_{443} E_{33}\right], \\
V_{S 1}^{2}= & \frac{1}{\rho_{0}}\left[C_{55}\left(1+2 E_{33}+2 E_{11}\right)+\sigma_{33}+C_{551} E_{11}\right. \\
& \left.+C_{552} E_{22}+C_{553} E_{33}\right] .
\end{aligned}
$$

The relative velocity difference expressions [Eqs. (4)-(6) in the text) are derived by using Eq. (33) to obtain linearized expressions for $V_{1}, V_{S 2}$, and $V_{S 3}$.

Thus, for example, 


$$
\begin{aligned}
& \frac{V_{1}-V_{i}^{\circ}}{V_{i}^{\circ}}=\frac{1}{4 C_{33}}\left[4 C_{33}\left(S_{13}+S_{23}\right)+C_{331}\left(S_{\mathrm{t} 1}+S_{12}\right)\right. \\
& \left.+C_{132}\left(S_{12}+S_{22}\right)+\sigma_{333}\left(S_{13}+S_{23}\right)\right]\left(\sigma_{1}+\sigma_{2}\right) \\
& +\frac{1}{4 C_{33}}\left[4 C_{33}\left(S_{13}-S_{23}\right)+C_{331}\left(S_{11}-S_{\mathrm{t} 2}\right)\right. \\
& \left.+C_{132}\left(S_{12}-S_{22}\right)+C_{333}\left(S_{13}-S_{23}\right)\right]\left(\sigma_{1}-\sigma_{2}\right)
\end{aligned}
$$

where $S_{i j}$ is the contracted matrix form of the compliance tensor $S_{i j k l}$. The coefficients of $\left(\sigma_{1}+\sigma_{2}\right)$ and $\left(\sigma_{1}-\sigma_{2}\right)$ are the constants $A$ and $D$ in Eq. (4). For an isotropic material $S_{12}=S_{13}=S_{23}, S_{11}=S_{12}$, and $C_{331}=C_{332}$ so $D=0$. For weak anisotropy, $D$ is of the order $\epsilon$, where $\epsilon$ is a small parameter characterizing the degree anisotropy (e.g., $\epsilon=C_{55}-C_{44}$ ). Similarly, Eqs. (5) and (6) in the text are obtained with $E$ and $F$ negligible for weak anisotropy.

Expressions for the square of velocities of $\mathrm{SH}$ waves propagating in the $X_{1}-X_{3}$ and $X_{2}-X_{3}$ planes are presented in the text [Eqs. (24) and (25)]. Velocity difference expressions are obtained from these by deriving linearized expressions for the velocities and introducing the compliance tensor to relate strain to stress. The result for the difference between the $\mathrm{SH}$-wave velocities in the $X_{2}-X_{3}$ and $X_{1}-X_{3}$ planes is

$$
\begin{aligned}
\left.\frac{\mathrm{SH}_{23}-\mathrm{SH}_{13}}{\mathrm{SH}^{\circ}}\right|_{\phi}= & \left.\frac{\mathrm{SH}_{23}{ }^{\circ}-\mathrm{SH}_{13}^{\circ}}{\mathrm{SH}^{\circ}}\right|_{\phi}-\frac{1}{2} \frac{a b}{(a+b)} \\
& \times\left[S(a-b)\left(2 C_{66} S 8+C_{661} S 5+C_{662} S 6+C_{663} S 7\right)-(a+b)\right] \\
& +C\left\{a\left[2 C_{44}\left(s_{11}\right]+s_{13}-s_{12}-s_{13}\right)+C_{551} S 5+C_{552} S 6+C_{553} S 7\right] \\
& -b\left[2 C_{44}\left(S_{12}+s_{13}-S_{22}-S_{23}\right)+C_{441} S 5+C_{442} S 6+C_{443} S 7\right]\left(\sigma_{2}-\sigma_{1}\right) \\
& +\frac{a b}{2} \frac{b}{(a+b)}\left[S ( a - b ) \left\{1+2 C_{66}\left(S_{11}+s_{22}+2 s_{12}\right)\right.\right. \\
& \left.+C_{661} S 2+C_{662} S 3+C_{663} S 4\right\}+C\left\{a \left[2 C_{55}\left(S_{11}+S 1\right)\right.\right. \\
& \left.\left.\left.+C_{551} S 2+C_{552} S 3+C_{553} S 4\right]-b\left[2 C_{44}\left(S_{22}+S 1\right)+C_{441} S 2+C_{442} S 3+C_{443} S 4\right]\right\}\right]\left(\sigma_{2}+\sigma_{1}\right),
\end{aligned}
$$

where $S=\sin ^{2} \phi, C=\cos ^{2} \phi, a=\frac{1}{2}\left[\rho_{0}\left(C_{66} s+C_{55} C\right)-\frac{1}{2}\right.$, $b=\frac{1}{2}\left[\rho_{0}\left(C_{66} s+C_{44} C\right)\right]-\frac{1}{2}, \quad S 1=s_{12}+s_{23}+s_{13}$, $S 2=s_{11}+s_{12}, \quad S 3=s_{12}+s_{22}, \quad S 4=s_{13}+s_{23}$, $S 5=s_{11}-s_{12}, \quad S 6=s_{12}-s_{22}, \quad S 7=s_{13}-s_{23}$, $S 8=s_{11}-S_{22}$.

The coefficients of $\sigma_{2}-\sigma_{1}$ and $\sigma_{2}+\sigma_{1}$ in Eq. (35) are the constants $a(\phi)$ and $c(\phi)$ presented in the text. For isotropic materials, $C_{55}=C_{44}, S_{11}=S_{22}, C_{551}=C_{442}, C_{441}=C_{552}$, and $C_{553}=C_{443}$, so $c(\phi)=0$.

For weak anisotropy, $c(\phi)$ is of order $(\epsilon)$ and may be neglected and Eq. (35) reduces to

$$
\left.\frac{\mathrm{SH}_{23}-\mathrm{SH}_{13}}{\mathrm{SH}^{\circ}}\right|_{\phi}=\frac{C_{44}-C_{55}}{2 C_{44}} \cos ^{2} \phi+a(\phi) ;
$$

$$
\begin{aligned}
a(\phi)= & \frac{1}{C_{44}}\left[\sin ^{2} \phi-\cos ^{2} \phi\left(S_{11}-S_{12}\right)\right. \\
& \left.\times\left(2 C_{55}+C_{551}-C_{552}\right)\right]+0(\epsilon) .
\end{aligned}
$$

In a similar manner Eq. (37) in the text is derived, and it is found $d(\phi)$ is negligible for weak anisotropy.

'D. S. Hughes and J. L. Kelly, Phys. Rev. 92, 1145 (1953).

${ }^{2}$ A. Seeger and O. Buck, Z. Naturforscher A15, 1056 (1960).

${ }^{3}$ R. N. Thurston and K. Brugger, Phys. Rev. A 133, 1604 (1965).

${ }^{4}$ R. N. Thruston, J. Acoust. Soc. Am. 37, 348 (1965).

${ }^{5}$ T. Tokuoka and M. Saito, J. Acoust. Soc. Am. 5, 1241 (1971).

${ }^{6}$ O. I. Gushcha and F. G. Makhort, Prikl. Mekh. 12, 32 (1976).
${ }^{7}$ E. G. Henneke II and R. E. Green, Jr., J. Acoust. Soc. Am. 45, 1367 (1969). ${ }^{8}$ H. Fukuoka, H. Toda, and T. Yamane, Exp. Mech. 18, 227 (1978).

${ }^{9}$ N. N. Hsu, Exp. Mech. 14, 169 (1974).

${ }^{10} \mathrm{E}$. Schneider, K. Goebbels, and G. Hubschen, Rev. Cethedec 17, 155 (1980).

"M. James and O. Buck, CRC Crit. Rev. Solid State Mater. Sci. 9, 61 (1980).

${ }^{12}$ G. S. Kino, J. B. Hunter, G. C. Johnson, A. R. Selfridge, D. M. Barnett. G. Hermann, and C. R. Steele, J. Appl. Phys. 50, 2607 (1979).

${ }^{13}$ M. P. Scott, D. M. Barnett, and D. B. Ilic, in 1979 Ulirasonics Symposium Proceedings, edited by J. de Klerk and B. R. McAvoy (IEEE, New York. 1980), p. 265.

${ }^{14}$ A. N. Gux, F. G. Makhort, O. I. Gushcha, and V. K. Lebedev, Prik1. Mekh. 7, 120S (1971).

${ }^{15}$ L. Niklas, Material Prufung 7, 281 (1965).

${ }^{16}$ T. Tokuoka and Y. I washimizu, Int. J. Solids Struct. 9, 383 (1968).

${ }^{17}$ K. Brugger, Phys. Rev. A133, 1611 (1964).

${ }^{18}$ Computer Code EIGRS (International Mathematical and Statistical L1braries, Houston, Texas, 1980).

${ }^{19}$ R. Bechmann, R. F. Hearmon, and S. K. Kurtz, Elastic, Piezoelectric and Related Constants of Crystals (Springer, Berlin, 1969), Vol. 2, pp. 102-123.

${ }^{20}$ D. I. Crecraft, J. Sound Vib. 5, 173 (1967).

${ }^{21}$ G. C. Johnson, Doctoral Dissertation, Stanford University, (1979).

${ }^{22}$ M. P. Scott, Doctoral Dissertation, Stanford University, 1981

${ }^{23}$ C. M. Fortunko, R. B. King, and N. Tan, J. A ppl. Phys. 53. 3450 (1982).

${ }^{24}$ C. F. Vasile and R. B. Thompson, J. Appl. Phys. 50, 2853 (1979).

${ }^{25}$ D. B. Ilic, G. S. Kino, A. R. Selfridge, and F. R. Stanke, in 1979 Ulirasonics Symposium Proceedings, edited by J. deKerk and B. R. McAvoy (IEEE, New York, 1980), p. 269.

${ }^{26}$ H. J. McSkimin, J. Acoust. Soc. Am. 35, 490 (1963).

${ }^{27}$ E. P. Papadakis, J. Acoust. Soc. Am. 40, 863 (1966).

${ }^{28}$ W. F. Pardee and R. B. Thompson, J. Nondesir. Eval. 1, 157 (1980) 

ARBITRARY PLANE RESIDUAL-STRESS

MEASUREMENTS (1983) 



\title{
Acoustoelastic evaluation of arbitrary plane residual stress states in non- homogeneous, anisotropic plates
}

\author{
R.B. KING and C.M. FORTUNKO
}

In this paper it is shown that relative ultrasonic velocity measurements can be used to determine the difference of normal stress components $\left(T_{22}-T_{11}\right)$ in non-homogeneous, anisotropic plates containing arbitrary residual stress states. It has previously been demonstrated that measurements with horizontally polarized shear waves (SH-waves) can be used to determine $\left(T_{22}-T_{11}\right)$ unambiguously in plates with unknown material property variations. However, previously the theory relating the velocity of $\mathrm{SH}$-waves to stress and material anisotropy was limited to the case where the principal directions of stress are parallel to the axes of material symmetry. In this paper, we remove this restriction by extending the theory. We also suggest possible experimental procedures for validating the new theory.

KEYWORDS: ultrasonics, stress measurement, anisotropic plates

\section{Introduction}

In a previous publication, we have addressed the problem of measuring two-dimensional residual stress distributions in non-homogeneous. anisotropic metal polycrystals ${ }^{1}$. By non-homogeneous we mean that the material properties vary with position. resulting in a variation of the wave speeds in the unstressed state. Specifically. we have developed a special theory of acoustoelasticity to describe the effects of in-plane residual stress states in plates on the velocities of shear waves polarized parallel to the surfaces and propagating obliquely with respect to the surface normal (SH-waves). To develop the new theory. we have assumed that the axes of symmetry of the textured. polycrystalline aggregate coincide with the principal stress directions. This assumption was needed to obtain simple. closed-form expressions describing the effects of stress and elastic anisotropy on the velocities of $\mathrm{SH}$ waves in the planes of symmetry.

We verified the theory using a new experimental procedure for evaluating the difference of the two inplane principal stress components. $\left(T_{22}-T_{11}\right)$, in the coordinate system of Fig. 1. ( $T_{i j}$ is the stress tensor.)

In this paper. we extend the new theory to the case of a more general stress state in a plate. In particular. we treat the case where the in-plane principal stress directions are not coincident with the in-plane material coordinates. as indicated in Fig. 1.

\section{Method}

In Ref. 1. we derived the following expression for the relative velocity of $\mathrm{SH}$-waves

The authors are in the Fracture and Deformation Division, National Bureau of Standards. Boulder, Colorado B0303, USA. Paper received 13 June 1983. Contribution of the National Bureau of Standards, not subject to copyright.

$$
\begin{aligned}
& \left.\frac{S H_{23}-S H_{13}}{S H_{0}}\right|_{\theta}=\frac{C_{55}-C_{44}}{C_{44}} \times \\
& \cos ^{2} \theta+a(\theta)\left(T_{22}-T_{11}\right)
\end{aligned}
$$

where $S H_{i j}$ denotes the velocity of a plane $\mathrm{SH}$-wave that propagates in the $x_{1}-x_{j}$ plane. as defined in Fig. 1 the angle $\theta$ is the acute angle between the direction of propagation and the normal to the surface: $C_{11}$ is the elastic constant tensor in contracted (Voigt) notation: and $a(\theta)$ is a calibration constant that is determined by the second and third-order elastic constants.

In deriving ( 1 ) we made the following assumptions: 1 the principal stress directions and material symmetry axes coincide, and 2 . only plane stress is present. It should be noted that coincidence of material symmetry axes and principal stress directions occurs only when

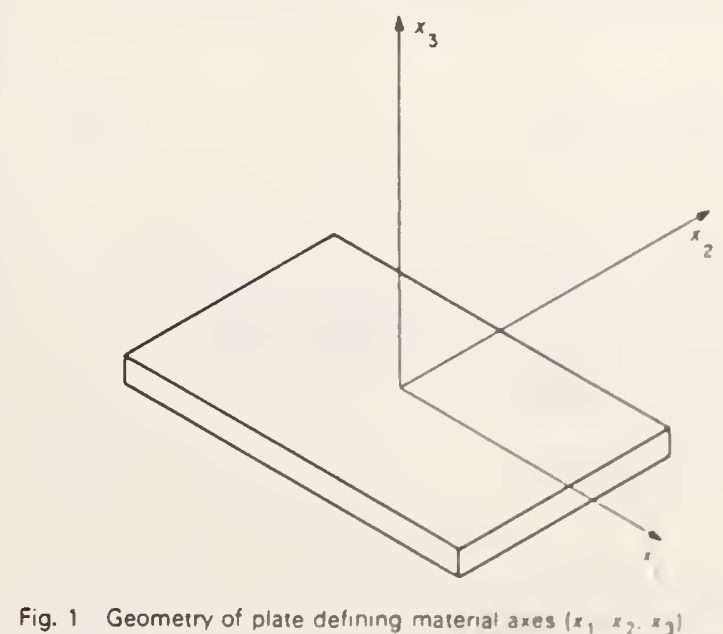


$T_{12}$ is zero. As a consequence of the second assumption, the stress components $T_{13}, T_{23}$, and $T_{33}$ vanish.

For the special case of grazing incidence $(\theta=\pi / 2)$, the anisotropy term in (1) vanishes. As a result. it can be rewritten as

$$
\left.\frac{S H_{23}-S H_{13}}{S H_{0}}\right|_{\pi / 2}=a(\pi / 2)\left(T_{22}-T_{11}\right)
$$

where

$$
a(\pi / 2)=1 /\left(2 C_{66}\right)
$$

The special result of (2), which holds for plane $\mathrm{SH}$ waves propagating at grazing incidence. has been proposed as the basis for evaluating residual stress states in plate-like geometries by Thompson et $\mathrm{al}^{2}$. However, the usefulness of the result is restricted by the assumption that the material symmetry axes and principal stress directions coincide. In this section. we extend the theory to include more general cases of stress distributions in plate-like geometries.

We follow the general theory of acoustoelasticity developed by Tokuoka and Iwashimizu ${ }^{3}$. Thus, the propagation of a plane wave in a stressed continuum is described by the following eigenvalue relationships

$$
\left[S_{i j k l} p_{i} p_{l}+\left(T_{i l} p_{i} p_{l}-\rho \nu^{2}\right) \delta_{j k}\right] d_{k}=0
$$

where $p_{i}$ is the unit normal to the wavefront. $d_{k}$ is a unit vector in the direction of the particle motion. $\rho$ is the material density, $v$ is the wave phase velocity. and

$$
\begin{aligned}
& S_{\dot{j} k l}=\rho / \rho_{0}\left(C_{m n p q}+C_{m n p q r s} E_{r s}\right) \\
& \frac{\partial x_{i}}{\partial X_{m}} \frac{\partial x_{j}}{\partial X_{n}} \frac{\partial x_{k}}{\partial X_{p}} \frac{\partial x_{l}}{\partial X_{q}}
\end{aligned}
$$

where $\rho_{0}$ denotes the material density in the unstressed state; $x_{i}$ and $X_{i}$ are the position vectors in the stressed and unstressed states respectively: $E_{i j}$ is the strain tensor; and $C_{i j k l}$ and $C_{i j k l m n}$ are the second and third order stiffness tensors. We assume the tensors $C_{i j k l}$ and $C_{i j k l m n}$ exhibit orthorhombic symmetry. (The general symmetry properties of $C_{i j k l m n}$ are described in Ref. 4.) A summation from 1 to 3 is implied when the indices are repeated. To evaluate (4), we find it convenient to introduce the relation

$$
\frac{\partial x_{i}}{\partial X_{j}}=\left(\delta_{i j}+E_{i j}+W_{i j}\right)
$$

where $W_{i j}$ is the local rigid body rotation tensor.

We first consider the case of a plane SH-wave propagation in the $x_{2}-x_{3}$ plane. In this case, $p_{1}=0$. $p_{2}=\sin \theta$, and $p_{3}=\cos \theta$. We rewrite (3) using a simplified matrix notation

$$
\left[A_{j k}-\rho_{0} \nu^{2} \delta_{i k}\right] d_{k}=0
$$

where

$$
A_{j k}=\left(S_{i j k l} p_{i} p_{l}+T_{i l} p_{i} p_{l} \delta_{j k}\right)
$$

From an expansion of (6) we obtain

$$
\begin{aligned}
& \left(A_{11}-\rho_{0} v^{2}\right)\left[\left(A_{22}-\rho_{0} v^{2}\right)\left(A_{33}-\rho_{0} v^{2}\right)-A^{2}{ }_{23}\right] \\
& -A_{12}\left[A_{12}\left(A_{33}-\rho_{0} v^{2}\right)-A_{23} A_{13}\right] \\
& +A_{13}\left[A_{12} A_{23}-\left(A_{22}-\rho_{0} v^{2}\right) A_{13}\right]=0
\end{aligned}
$$

Next. from (3). (4), and (7) we obtain the following

$$
\begin{aligned}
A_{12} & =\left[\left(C_{12}+C_{22}+2 C_{66}+C_{266}\right) E_{12}\right. \\
& \left.+\left(C_{22}-C_{12}-2 C_{66}\right) W_{12}\right] \sin ^{2} \theta \\
& +\left[\left(C_{44}-C_{95}+C_{456}\right) E_{12}+\left(C_{44}-C_{55}\right) W_{12}\right] \cos ^{2} \theta
\end{aligned}
$$

and

$$
\begin{aligned}
& A_{13}=2 \sin \theta \cos \theta\left[\left(C_{13}+C_{23}+C_{366}\right) E_{12}\right. \\
& \left.\quad+\left(C_{23}-C_{13}\right) W_{12}\right]
\end{aligned}
$$

Noting that $A_{12}$ and $A_{13}$ are of one order higher than $A_{11}, A_{22}$, and $A_{33}$ in the displacement gradients. we obtain the following approximation to (8)

$$
\left(A_{11}-\rho_{0} v^{2}\right)\left(A_{22}-\rho_{0} v^{2}\right)\left(A_{33}-\rho_{0} v^{2}\right)=0
$$

The solution corresponding to the SH-wave is

$$
\rho_{0} v^{2}=A_{11}
$$

Now, we introduce the notation $S H_{i j}$ to denote the velocity of an SH-wave in the $x_{i}-x_{j}$ plane. From (3). (4) and (7) we obtain in the $x_{2}-x_{3}$ plane

$$
\begin{aligned}
& \rho_{0} S H^{2}{ }_{23}=\left[C_{66}\left(1+2 E_{11}+2 E_{22}\right)+C_{66 i} E_{i}+T_{22}\right] \sin ^{2} \theta \\
& +\left[C_{55}\left(1+2 E_{11}+2 E_{33}\right)+C_{55 i} E_{i}+T_{33}\right] \cos ^{2} \theta
\end{aligned}
$$

Similarly, in the $x_{1}-x_{3}$ plane we obtain

$$
\begin{aligned}
& \rho_{0} S H^{2}{ }_{13}=\left[C_{66}\left(1+2 E_{11} 2 E_{22}\right)+C_{66 i} E_{i}+T_{11}\right] \sin ^{2} \theta \\
& +\left(C_{44}\left(1+2 E_{33}+2 E_{22}\right)+C_{44 i} E_{i}+T_{33}\right] \cos ^{2} \theta
\end{aligned}
$$

\section{Conclusion}

Equations 13 and 14 are identical to those presented in Ref. 1 for the special case $T_{12}=0$. Thus. to first order in displacement gradients, the presence of non-zero $T_{12}$ produces no effect on the velocities of $\mathrm{SH}$-waves propagating in the planes of material symmetry.

To obtain an expression for experimental evaluation. we developed a linearized velocity difference equation from (13) and (14). In addition we assumed weak anisotropy and expressed strain in terms of stresses using Hooke's law. After algebraic simplification we obtained

$$
\begin{aligned}
& \left(S H_{23}-S H_{13}\right) / S H_{0}=\cos ^{2} \theta\left(C_{55}-C_{44}\right) / 2 C_{66} \\
& +\left(T_{22}-T_{11}\right)\left[\sin ^{2} \theta-\cos ^{2} \theta\left(C_{441}-C_{551}\right) / 2 C_{66}\right] / 2 C_{66}
\end{aligned}
$$




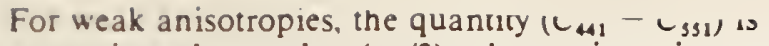
approximately equal to $(-n / 2)$, where $n$ is an isotropic Mumaghan constant. Equation (15) is identical to (1), with $a(\theta)$ explicitly given by

$$
a(\theta)=\left|\sin ^{2} \theta-\cos ^{2} \theta n / 4 C_{66}\right| / 2 C_{66}
$$

Funhermore, an error magnification effect that limits the precision of stress evaluation with obliquely incident waves' is not present for grazing SH-waves. However. to our knowledge an accurate experimental procedure based on the use of grazing SH-waves has not yet been experimentally demonstrated.

The authors have learned by private communication that a similar theory to that presented in this note has been derived by A.V. Clark. He has investigated the more general case where the stress components $T_{13}$ and
$1{ }_{23}$ are non-vanishing and has also derived an interesting restriction on the theory leading to (15) for $\mathrm{SH}$-waves approaching normal incidence.

\section{Acknowledgements}

This work was supported by the National Bureau of Standards, Office of Nondestructive Evaluation. The authors wish to express their appreciation to JoAnne Wilken for typing the manuscript.

\section{References}

1 King, R.B., Fortunko, C.M. J. Appl Phys. in press

2 Thompson, R.B., Smith, J.F., Lee, S.S. unpublished.

3 Tokuoke, T., Iwashimizu, Y. Int J. Solids Structures. 9 (1968) 383

4 Bechmann, R., Hearmon, R.F., Kurtz, S.K. Elastic

Piezoelectric. and Related Constants of Crystals SpringerVerlag. Berlin (1969) 2. 102-123 
Evaluation of Surface Residual Stresses Using Horizontally Polarized Shear Waves At Grazing Incidence

\author{
R.B. King and C.M. Fortunko \\ Fracture and Deformation Division \\ National Bureau of Standards \\ Boulder, Colorado 80303
}

to be published by: Journal of Applied Physics

Keywords: acoustoelasticity; anisotropy; elastic waves; geometrical acoustics; nondestructive evaluation; residual stress.

Contributions of National Bureau of Standards are not subject to copyright in the U.S. 


\section{ABSTRACT}

In this paper a new theory and experimental method are described for evaluation of surface residual stresses in inhomogeneous, anisotropic materials. The method is based on the use of horizontally polarized shear waves (SH-waves) that propagate at a grazing angle with respect to the surface of a sample. In addition, a new theory is presented for grazing SH-waves propagating through a body in which the stress distribution varies with depth. It is shown that to first order the average velocity of the grazing SH-waves is dependent only on the surface values of residual stress. Based on the use of electromagnetic-acoustic transducers (EMATs) to generate and detect grazing SH-waves, preliminary experimental verification of the theory is presented. 
INTRODUCTION

It is well known that ultrasonic determination of residual stress is fundamentally complicated by texture and microstructure effects. Texture and microstructure can cause the material to be anisotropic, and variations in texture and microstructure lead to material inhomogeneity. Anisotropy and inhomogeneity influence ultrasonic wave propagation and, often, mask the stress related effects. In a previous paper [1], the authors have demonstrated that conventional ultrasonic methods, which rely on bulk shear or longitudinal waves at normal incidence with respect to a free surface, cannot provide enough information to separate the effects of stress and material properties on ultrasonic wave velocities. Techniques based on changes in the Rayleigh wave velocities are also sensitive to limitations caused by material property variations. In Ref. 1, the authors have described a new ultrasonic measurement technique that can be used to overcome the above limitations. To verify the new technique, the authors have compared ultrasonically-determined stress distributions in a plate with theoretical predictions and strain gage measurements. Good quantitative agreement has been demonstrated.

In contrast to the conventional ultrasonic techniques, the technique proposed by the authors in Ref. 1 is based on the use of shear waves that are polarized parallel to and propagate obliquely with respect to the free surface. These special ultrasonic probing waves are SH-waves, which can now be efficiently generated and detected using electromagnetic-acoustic transducers (EMATS) [1].

The new ultrasonic technique described by the authors in Ref. 1 is limited in two respects. First, the theoretical basis for the technique was limited to cases when the stresses coincided with the material coordinate axes. This limitation was later removed, however, by extending the theory. The 
modified theory is presented in Ref. 2. A second limitation involves variations of stress in the direction normal to the free surfaces of a sample. When such variations are present, the average through-thickness value of stress is determined. Thus, stress distributions which have a vanishing average through thickness value (for example, bending stresses) are not detected. This limitation is shared by all ultrasonic techniques that involve through-thickness propagation of the ultrasonic probing signals.

In situations where the residual stress varies in the thickness direction, the value of stress at the free surface is often of greatest importance. Consequently, this paper specifically addresses the problem of determining the surface values of residual stresses. In particular, a practical experimental procedure is described that permits the determination of surface residual stresses in weakly inhomogeneous and anisotropic materials.

The new experimental procedure is based on the use of SH-waves that propagate at near-grazing angles with respect to the free surfaces. Thus, the new procedure is different from the Rayleigh wave procedures that have also been used to evaluate surface stresses $[3,4]$. The Rayleigh wave procedures are sensitive to material property variations. On the other hand, the new SH-wave procedure appears to be inherently insensitive to material property variations.

\section{THEORETICAL BACKGROUND}

Before describing the theory underlying the new experimental for evaluating surface residual stresses, it is useful to outline the shortcomings of the Rayleigh wave technique. The theoretical basis for the Rayleigh wave technique was first proposed by Hayes and Rivlin [5]. In Ref. 5, the following relationship was derived:

$$
\left(v-v_{0}\right) / v_{0}=a T_{1}+b T_{2}
$$


where $v$ and $v_{0}$ denote the velocity of a Rayleigh wave in the unstressed and stressed states, respectively, $a$ and $b$ are functions of the second- and third-order elastic constants of the material, and $T_{1}$ and $T_{2}$ are the two nonvanishing principal stress components at the free surface. In Eq. 1 the axes of material symmetry and principal stress directions are taken to be paralle1. The coordinate system relevant to Eq. 1 is shown in Fig. 1 .

A linear combination of $T_{1}$ and $T_{2}$ can be inferred by making the relative velocity measurement needed to evaluate the left-hand-side of Eq. 1. However for many engineering materials, the value of $v_{0}$ is not constant with position. This effect causes a large uncertainty in the ultrasonically determined value of $\left(a T_{1}+b T_{2}\right)$. This particular problem can be overcome by making use of $\mathrm{SH}$-waves that propagate at near-grazing incidence.

The advantages of using SH-wave for evaluating stress in the presence of material property variations were first demonstrated in Ref. 1. In particular, based on the theory presented in Ref. 1, the following relation can be derived for SH-wave propagating at near-grazing incidence:

$$
\left(v_{2}-v_{1}\right) / v_{0}=\left[1 /\left(2 C_{66}\right)\right]\left(T_{22}-T_{11}\right)
$$

where, $v_{2}, v_{1}$ are the velocities of grazing SH-waves propagating in the $x_{2}$, $x_{1}$, directions, respectively, $v_{0}$ is the mean unstressed shear wave velocity, $T_{22}$ and $T_{11}$ are normal stress components referred to the coordinate system of Fig. 1, and $\mathrm{C}_{66}$ is an elastic constant. Equation 2 was independently proposed as the basis for evaluating bulk residual stresses by Thompson, Smith, and Lee $[6]$.

It is important to note that no unstressed velocity term appears in Eq.2. In particular, there is no difference between $v_{1}$ and $v_{2}$ in the unstressed state even though the theory is valid for orthorhombic materials. Thus, from a relative measurement of velocities in the unstressed state alone, the 
left-hand-side of Eq. 2 can be evaluated, and the quantity $\left(T_{22}-T_{11}\right)$ can be inferred. The theory used in deriving Eq. 2 is valid only for the case where the material properties (elastic constants and density) and stresses are homogeneous. In addition, in deriving Eq. 2, the assumption was made that the principal directions of stress and the material symmetry axes are parallel. This is equivalent to the assumption that $T_{12}$ vanishes in the coordinate system of Fig. 1 [1]. This restriction was later removed by the authors in Ref. 2, where the theory was extended to the case of arbitrary values of $T_{12}$. In this paper, the theory of Ref. [i] is extended to the case of neargrazing SH-wave propagating through inhomogeneous stress distributions. The elastic constants and the density of the material are taken to be constant in the region of interest, while the stress components are functions of $x_{3}$ only. This permits the study of a situation of practical importance. The problem of evaluating residual stresses in plates can be considered as an example. In plates, the stress distribution can vary over a short length scale in the thickness direction. The variation of stress with in-plane dimensions is over a longer distance, and can be neglected in the region of interest (the region interrogated experimentaliy).

Following Tokuoka and Iwashimizu [7], the equation of motion for a small displacement superimposed on a large elastic initial strain state is derived:

$$
\frac{\partial}{\partial x_{i}}\left\{\left(S_{i j k \ell}+T_{i \ell}\right) \frac{\partial u_{k}}{\partial x_{\ell}}-\rho \frac{\partial^{2} u_{j}}{\partial t^{2}}=0\right.
$$

where

$$
S_{i j k \ell}=\frac{\rho}{\rho}\left(C_{m n p q}+C_{m n p q r s} E_{r s}\right) \frac{\partial x_{i}}{\partial x_{m}} \frac{\partial x_{j}}{\partial x_{n}} \frac{\partial x_{k}}{\partial x_{p}} \frac{\partial x_{\ell}}{\partial x_{q}}
$$


$\rho_{0}, \rho$ are the densities in the unstressed and stressed states, respectively, $C_{\text {mnpq }}$ and $C_{\text {mnpqrs }}$ are the second- and third-order elastic tensors of the material, $E_{i j}$ is the strain tensor, $u_{i}$ is the displacement vector describing the small superimposed motion, and $x_{i}, x_{i}$ represent the position vectors of a particle in the unstressed and stressed states, respectively. The deformation gradients can be replaced using the relation (valid to first order in displacement gradients)

$$
\frac{\partial x_{i}}{\partial x_{j}}=\left(\delta_{i j}+E_{i j}+w_{i j}\right)
$$

where $W_{i j}$ is the local rigid body rotation. Equations 3 and 4 are valid for any small elastic displacements superimposed on an initial elastic strain state. The special case of an SH-wave propagating in the $x_{1}-x_{3}$ plane is treated first. In this case:

$$
u_{i}=u_{3}=0, u_{2}=u_{2}\left(x_{1}, x_{3}, t\right)
$$

Substituting the above in Eq. 3, the following result is obtained:

$$
\frac{\partial}{\partial x_{1}}\left(\alpha\left(x_{1}, x_{3}\right) \frac{\partial u_{2}}{\partial x_{1}}+\frac{\partial}{\partial x_{3}}\left(\beta\left(x_{1}, x_{3}\right) \frac{\partial u_{2}}{\partial x_{1}}-\rho_{0} \frac{\partial^{2} u_{2}}{\partial t^{2}}=0\right.\right.
$$

Henceforth the subscript "2" will be omitted. The functions $\alpha$ and $\beta$ depend on the elastic constants and strains and are presented in the Appendix.

It is noted that in Eq. 5 the functions $\alpha$ and $\beta$ are only slightly perturbed from homogeneous, isotropic values. Thus, they can be expressed as

$$
\alpha=C_{66}+\varepsilon f\left(x_{1}, x_{3}\right), B=C_{66}+\varepsilon g\left(x_{1}, x_{3}\right)
$$

where $\varepsilon$ is a small parameter. (Strain and degree of anisotropy are assumed to be of the same order of magnitude in this analysis. The value of $\varepsilon$ is of the 
order of strain). Thus, a direct perturbation solution in terms of $\varepsilon$ is possible. It can be shown, however, that this approach does not lead to a simple closed-form expression for $u$. In this paper, a geometrical acoustics approach is used instead. Following Karal and Keller [8], let

$$
u=A\left(x_{1}, x_{3}\right) \exp \left[i \omega\left(S\left(x_{1}, x_{3}\right)-t\right)\right]
$$

The amplitude $A$ is expanded in reciprocal powers of frequency,

$$
A=\sum_{n=0}^{\infty} A_{n}(1 / \omega)^{n}
$$

Substituting Eqs. 7 and 8 into Eq. 5, the zero'th order solution (valid in the high-frequency limit) is

$$
\text { Ao }\left[-\alpha\left(\frac{\partial S}{\partial x_{\bar{x}}}\right)^{2}-\beta\left(\frac{\partial S}{\partial x_{3}}\right)^{2}+\rho_{0}\right]=0
$$

For arbitrary $A_{0}$, there results

$$
\alpha\left(\frac{\partial S}{\partial x_{1}}\right)^{2}+\beta\left(\frac{\partial S}{\partial x_{3}}\right)^{2}=\rho_{0}
$$

Equation 10 is the Eikonal equation for SH-wave in an inhomogeneous, anisotropic medium. Solutions to Eq. 10 are cylindrical surfaces along which the phase, $S$, is constant (wavefronts). The family of curves that is orthogonal to the wavefronts is the family of rays. The phase, $S$, can be found by integrating forward along a ray from a known starting point. Equation 10 is not in convenient form because the functions $\alpha$ and $\beta$ are in general not equal. However, by a pertubation approach Eq. 10 can be converted to an isotropic 
equation with inhomogeneous right-hand-side. By substituting Eqs. 6 into Eq. 10 and moving terms involving $\varepsilon$ to the right-hand-side, one obtains:

$$
\left(\frac{\partial S}{\partial x_{1}}\right)^{2}+\left(\frac{\partial S^{2}}{\partial x_{3}}=\left\{0-\varepsilon f\left(\frac{\partial S^{2}}{\partial x_{1}}\right)-\varepsilon g\left(\frac{\partial S^{2}}{\partial x_{3}}\right\} / C_{66}=n^{2}\right.\right.
$$

where $n$ is the effective index of refraction of the medium. Equation 11 can be evaluated using an iterative procedure: First, $\varepsilon$ is set equal to zero to obtain $S_{0}$, the solution to zero'th order in $\varepsilon$. Then, $S_{0}$ is substituted into the right-hand-side of Eq. 11 and the first-order solution, $S_{1}$, is found. This procedure is used to study the case of a line source on the surface of a half-space (Fig. 2). The wavefronts in a homogeneous, anisotropic medium are circles defined by

$$
S_{0}=\left(p_{0} / C_{66}\right)^{1 / 2} R
$$

where $R$ is the radius measured from the line source. Substituting Eq. 12 into Eq. 11, for points in the vicinity if the surface, the approximate result is

$$
n=\left(\rho_{0} / C_{66}\right)^{1 / 2}\left(1-\varepsilon f / 2 C_{66}\right)
$$

If the stresses vary only in the $x_{3}$ direction, then $f=f\left(x_{3}\right)$, and from Eq. $13 n$ is a function of $x_{3}$ only. For isotropic Eikonal equations of the form of Eq. 11 , the rays are defined by $[8]$ :

$$
\frac{d}{d l}\left(n \frac{d x_{i}}{d l}\right)=\frac{d n}{d x_{1}}
$$

where $i$ takes on the values 1 and 3 and $\ell$ denotes arc length along a ray. 
The special case where the stresses vary linearly with $x_{3}$ is considered next. (This can be thought of as the first segment of a multilinear fit to an arbitrary stress distribution). In this case

$$
f=f_{0}+f_{1} x_{3}
$$

where $f_{0}$ and $f_{1}$ are constants. Equation 14 is evaluated to find the rays. Following Officer [9], the rays can be shown to be arcs of circles (Fig. 3) with centers along a line a distance

$$
\left[1-\varepsilon f_{0} /\left(2 C_{66}\right)\right] /\left[\varepsilon f_{1} /\left(2 C_{66}\right)\right]
$$

above the surface. Along a ray, the difference in phase, $S$, between two points $\ell_{0}$ and $\ell$ is [8]

$$
\Delta S=\int_{\ell 0}^{\ell} n d \ell
$$

If the source and observation points, both located along the free surface, are separated by a distance, $2 x$, as shown in Fig. 3 , then to first order in $\varepsilon$ the phase difference between the two points is given by

$$
\Delta S=2 \omega\left(C_{66} / \rho_{0}\right)^{1 / 2}\left[1-\varepsilon_{0} /\left(2 C_{66}\right)\right] x
$$

The phase difference in the unstressed medium is given by

$$
\Delta S_{0}=2 \omega\left(C_{66} / \rho_{0}\right)^{1 / 2} x
$$


From Eqs 15 and 6 and the definition of $\alpha$ in the Appendix, it is seen that $f_{0}$ depends only on the values of stress at the surface. Thus, to first order in strain the phase at the surface is only dependent on the stress values at the surface.

Equation 15 was derived for an SH-wave propagating in the $x_{1}-x_{3}$ plane. If a similar calculation is performed for a near-grazing SH-wave in the $x_{2}-x_{3}$ plane, an equivalent result to Eq. 15 is obtained. Calculating the relative phase difference between the near-grazing SH-waves in the $x_{1}-x_{3}$ and $x_{2}-x_{3}$ planes results in:

$$
\left(\Delta S^{2}-\Delta S^{1}\right) / \Delta S_{0}=-1 /\left(2 C_{66}\right)\left(T_{22}\left(x_{3}=0\right)-T_{11}\left(x_{3}=0\right)\right)
$$

where $\Delta S^{2}, \Delta S^{1}$ denote the phase difference between the source and observation points for the near-grazing SH-waves in the $x_{2}-x_{3}$ and $x_{1}-x_{3}$ planes. It is convenient to introduce the concept of average velocity along the arc connecting the source and observation points. If the frequency of the wave and the arc length between source and observation points are held constant while the plane of propagation is changed from the $x_{1}-x_{3}$ to the $x_{2}-x_{3}$ plane, then

$$
\left(\Delta s^{2}-\Delta s^{1}\right) / \Delta S_{0}=-\left(v_{2}-v_{1}\right) / v_{0}
$$

When Eq. 18 is substituted in Eq. 17, the result is

$$
\left(v_{2}-v_{1}\right) / v_{0}=1 /\left(2 C_{66}\right)\left[T_{22}\left(x_{3}=0\right)-T_{11}\left(x_{3}=0\right)\right]
$$

Thus, the relative velocity difference between a near-grazing SH-wave in the $x_{1}-x_{3}$ and $x_{2}-x_{3}$ planes is dependent only on the value of the quantity $\left(T_{22}-T_{11}\right)$ at the surface. This result forms the theoretical basis for a new experimental procedure for evaluating the surface values of residual stress in weakly inhomogeneous and anisotropic materials. 
Several comments are in order concerning the theory leading to Eq. 19. Equation 19 is valid to first order in strain. By keeping the next higher order term, the effects of stress gradients can be quantified. This is carried out in the Appendix, and it is shown that for realistic stress gradients, Eq. 19 provides results that are sufficiently accurate for engineering purposes.

It is interesting to note that the construction of Fig. 3 , with the line of centers of the rays above the surface, is valid only when the stress increases with depth. If the stress decreases with depth, the line of centers will be below the surface. Because the curvature of the rays is very slight, for the latter case, the source and observation points would have to be slightly below the surface. In practice, this is not a limitation since the transducers used to generate and receive SH-waves operate by producing and detecting body forces in the sample in a region slightly below the surface [1].

As a final note on the theory, Eq. 19 was derived using a geometrical acoustics approximation and thus is valid only in the high-frequency limit. However, keeping higher order terms in the asymptotic expansion (Eq. 8) affects only the amplitude and not the phase. Therefore, Eq. 19 is valid for lower frequencies as well. From physical considerations, the theory is likely to become invalid when the length scale over which significant stress variations occurs becomes comparable to or less than the ultrasonic wavelength.

\section{EXPERIMENTAL PROCEDURE AND RESULTS}

Recent developments have made it possible to construct efficient transducers of SH-waves, that employ an electromagnetic-acoustic transduction principle. The principle and operation of electromagnetic-acoustic transducers (EMATs) is described in Ref. 10. A typical transducer construction is shown in Fig. 4. 
The radiation pattern of the EMAT in Fig. 4 in the plane normal to the surface and containing the source and observation points is shown in Fig. 5 . It is seen from Fig. 5 that indeed a grazing SH-wave can be generated by this EMAT.

In the present work, the velocity measurement system described in Ref. 1 was used to make relative velocity measurements with grazing SH-waves. The transducer arrangement shown schematically in Fig. 6 was used in the experiments, with the two transducers separated by a distance, $2 x$, equal to $35 \mathrm{~mm}$.

Preliminary experiments were conducted, to investigate the sensitivity of SH-waves to anisotropy, and to validate the near-grazing SH-waves that propagate in an inhomogeneous stress field. The plate specimen shown in Fig. 7 was used in the experiments. The plate was made of aluminum 6061-T6 and was $25 \mathrm{~mm}$ thick.

In the first experiment, the anisotropy was characterized by making measurements with shear waves at a steep angle of incidence along the line shown in Fig. 7. The average value of velocity anisotropy obtained from several measurements in the unstressed sample was $0.005 \pm 12$ percent. On the other hand, the velocity difference $\left(v_{2}-v_{1}\right) / v_{0}$ of grazing SH-waves in the same unstressed sample averaged 0.00011 from several measurements. The effect of anisotropy is seen to be substantially smaller along a grazing path than along a steep path, by a factor of almost 50. This result confirms the prediction of the theory (Eq. 1).

A calibration test was next performed by placing the specimen shown in Fig. 7 in a tensile testing machine. A maximum tensile stress of $210 \mathrm{MPa}$ was applied. Relative velocity measurements, $\left(v_{2}-v_{1}\right) / v_{0}$, were made at various stress levels. From the calibration test and Eq. 2, the result for $\mathrm{C}_{66}$ was $2.63 \times 10^{9} \mathrm{MPa}$. As expected, this does not differ significantly from the 
nominal value of the shear modulus for aluminum 6061-76 of $2.66 \times 10^{4} \mathrm{MPa}$ [11] The specimen shown in Fig. 7 was placed in a four point bending apparatus. A load of $30 \mathrm{kN}$ was applied, giving rise to a maximum stress of $226 \mathrm{MPa}$ at the surface and a stress distribution that varies linearly through the thickness in accordance with beam theory. (The stress was constant along the beam in the vicinity of the middle of the specimen). Several relative velocity measurements were made at different points in the region near the center of the specimen. The resulting average value for $\left(v_{2}-v_{1}\right) / v_{0}$ was $0.0045 \pm 15 \%$. Using the calibration result for $\mathrm{C}_{66}$, the estimate of the surface value of $\left(\mathrm{T}_{22}{ }^{-\mathrm{T}} 11\right)$ from the velocity measurements using Eq. 19 was $236 \mathrm{MPa} \pm 15^{\circ} \%$. The discrepancy of the average value from the correct value of $226 \mathrm{MPa}$ is only $5 \%$. (It is expected that better precision and reproducibility can be obtained with improved transducers). The above experimental results are in agreement with the new theory.

\section{Discussion}

Based on Eq. 19, relative velocity measurements with grazing $\mathrm{SH}$-waves can be used to evaluate the surface value of $\left(T_{22}{ }^{-T} 11\right)$ in inhomogeneous, anisotropic materials subjected to residual stress. This technique has two distinct advantages over the use of other ultrasonic procedures such as measurements with Rayleigh waves. The first is that no unstressed velocity term appears in Eq. 19, the basis for the new procedure, while $\left(v_{2}-v_{1}\right) / v_{0}$ does appear in Eq. 1, the basis for the Rayleigh wave approach. Slight material inhomogeneities can cause variations in $v_{0}$ which are of the same order as the small change in $v$ caused by stress. Thus, severe errors would result in the evaluation of stress from Rayleigh wave measurements and Eq. 1. This is not a problem with the new procedure proposed in this paper. A second advantage is 
that the coefficient relating stress to velocity (henceforth referred to as an acoustoelastic constant) in Eq. 19 is only dependent on one second-order elastic constant, $C_{66}$. In contrast, the coefficients a and $b$ in $E q .1$ are functions of the second-and third-order elastic constants of the material. Similarly, the coefficients relating velocity to stress for techniques based on through-thickness propagation of bulk waves are also dependent on both second-and third-order elastic constants. In typical engineering materials, slight inhomogeneities are likely to cause a stronger effect on the secondorder elastic constants than on the third-order elastic constants. For example, Hunter [12] reported results comparing properties of specimens, that had nominally identical compositions but undergone different forming operations. The Young's modulus and shear modulus are similar for the specimens of the two materials. However, the acoustoelastic constant for bulk longitudinal waves differed by almost $50^{\circ} / 0$ between the specimens made of the two materials. Johnson [13] explained this result by showing that texture, which is different in Aluminum 6061-T6 bars than in Aluminum 6061-T651 plates, has a stronger effect on third-order elastic constants than on second-order elastic constants.

The variation of the acoustoelastic constant with position will cause errors in the evaluation of stress from velocity measurements if an average value of the acoustoelastic constant has been used which has been calibrated using a separate reference sample of the material under study. Scott [14] proposed an in-situ calibration technique that permits calibration of the acoustoelastic constant as a function of position directly in the residual stress sample of interest. However, this technique requires a means of superimposing an additional elastic stress state on the residual stress state in the sample. Therefore this procedure may be inconvenient in practical 
configurations. An in-situ calibration technique is less likely to be necessary for the procedure presented in this paper because the calibration constant is likely to vary less strongly with position.

The effective spatial resolution of the new procedure is determined by the spacing between the transmitter and receiver transducers. The lower limit of the spacing is controlled by the decay time of the initial electromagnetic drive pulse (currently about 10 microseconds), corresponding to approximately $30 \mathrm{~mm}$. 


\section{Appendix}

Explicit expressions for the functions $\alpha$ and $\beta$ discussed in the text are as follows:

$$
\begin{aligned}
& \alpha=C_{66}\left(1+E_{1}+E_{2}-E_{3}\right)+C_{66 i} E_{i}+C_{11} E_{1}+C_{12} E_{2}+C_{13} E_{3} \\
& B=C_{44}\left(1+E_{2}+E_{3}-E_{1}\right)+C_{44 i} E_{i}+C_{13} E_{1}+C_{23} E_{2}+C_{33} E_{3}
\end{aligned}
$$

In Eqs. 21 and 22, contracted notation has been used for both the stiffness and strain tensors.

Keeping the next higher order term, the result for the phase difference between source and observation points for a grazing SH-wave propagating in the

$$
\begin{aligned}
& x_{2}-x_{3} \text { plane is } \\
& \quad S=\left(\rho_{0} / C_{66}\right)^{1 / 2}\left[1-\varepsilon f_{0} /\left(2 C_{66}\right)\right] x-\left[\varepsilon f_{1} /\left(3 C_{66}\right)\right]\left(\rho_{0} / C_{66}\right)^{1 / 2} x^{2} \varepsilon
\end{aligned}
$$

A realistic residual stress distribution in a plate is shown in Fig. 8 . For a source-to-observation point spacing equal to $h$, the thickness of a plate, based on the distribution in Fig. 8, the error introduced by neglecting the second term in Eq. 23 is less than 8 percent. Because this technique eliminates the potentially large errors in other approaches for evaluating surface values as residual stress (e.g. the use of Rayleigh waves) caused by unknown material property variations, the error level of 8 percent can be considered acceptable for engineering purposes. (The error is reduced as the source-to-observation point spacing is reduced). 
Acknowledgments

This work was supported by the U. S. Navy, David Taylor Naval Ship Research and Development Laboratory, and the National Bureau of Standards, Office of Nondestructive Evaluation. The authors wish to express their appreciation to JoAnne Wilken for typing the manuscript. The assistance of John Moulder and John Arvidson, Jr. in constructing the special EMATs used and obtaining experimental data is also gratefully acknowledged. 


\section{REFERENCES}

[1]. R.B. King and C.M. Fortunko, J. Appl. Phys., in press.

[2]. R.B. King and C.M. Fortunko, unpublished.

[3]. M. Hirao, H. Fukuoka, and K. Hori, J. Appl. Mechanics 48, 119 (1981).

[4]. D. Husson, S.D. Bennett, and G.S. Kino, Proceedings, 1982 IEEE UTtrasonics Symposium, ed. by B. R. McAvoy (IEEE, New York 1982) pp. 889--892.

[5]. M. Hayes and R.S. Rivlin, Arch. Rat. Mech. Anal. 8, 358 (1961).

[6]. R.B. Thompson, J.F. Smith, and S.S. Lee, Proceedings, 1982 AF/DARPA Review of Progress in Quantitative NDE, ed. by D. O. Thompson and D. L. Chimenti (Plenum, New York, 1983).

[7]. T. Tokuoka and Y. Iwashimizu, Int. J. Solids and Structures 9,383 (1968).

[8]. F.C. Karal and J.B. Keller, J. Acoust. Soc. Am. 31,694 (1959).

[9]. C.B. Officer, Introduction to the Theory of Sound Transmission, (McGraw-Hi11, N.Y., 1958) pp. 59-61.

[10]. C.M. Fortunko and R.B. King, J. Appl. Phys. 53,3450 (1982).

[11]. A. Higdon, E. Ohlger, W. Stiles, J. Weese, Mechanics of Materials, (Wiley, N.Y., 1967) pp. 554-555.

[12]. J. B. Hunter, Ph.D. Dissertaiton, Mechanical Engineering Department, Stanford University, 1979, unpublished.

[13]. G. C. Johnson, Proceedings, 1982 AF/DARPA Review of Progressin Quantitative NDE, ed. D. 0. Thompson and D. L. Chimenti (Plenum, NY, 1983) in press.

[14]. M. P. Scott, Ph.D. Dissertation, Materials Science and Engineering Department, Stanford University, 1980, unpublished. 


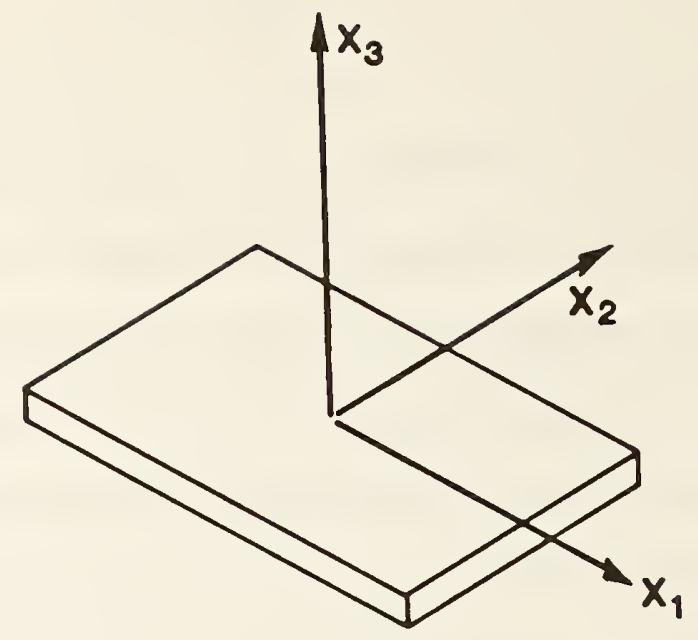

Fig. 1. Coordinate system used in derivations.

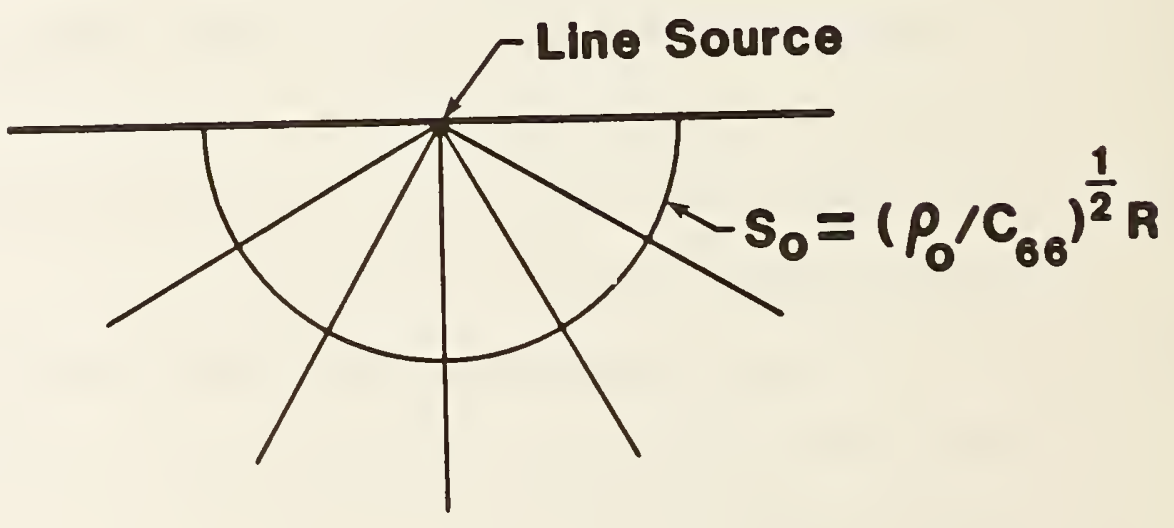

Fig. 2. Line source on surface of half-space. 


\section{Source Point}

Fig. 3. Rays in vicinity of surface for constant velocity gradient.

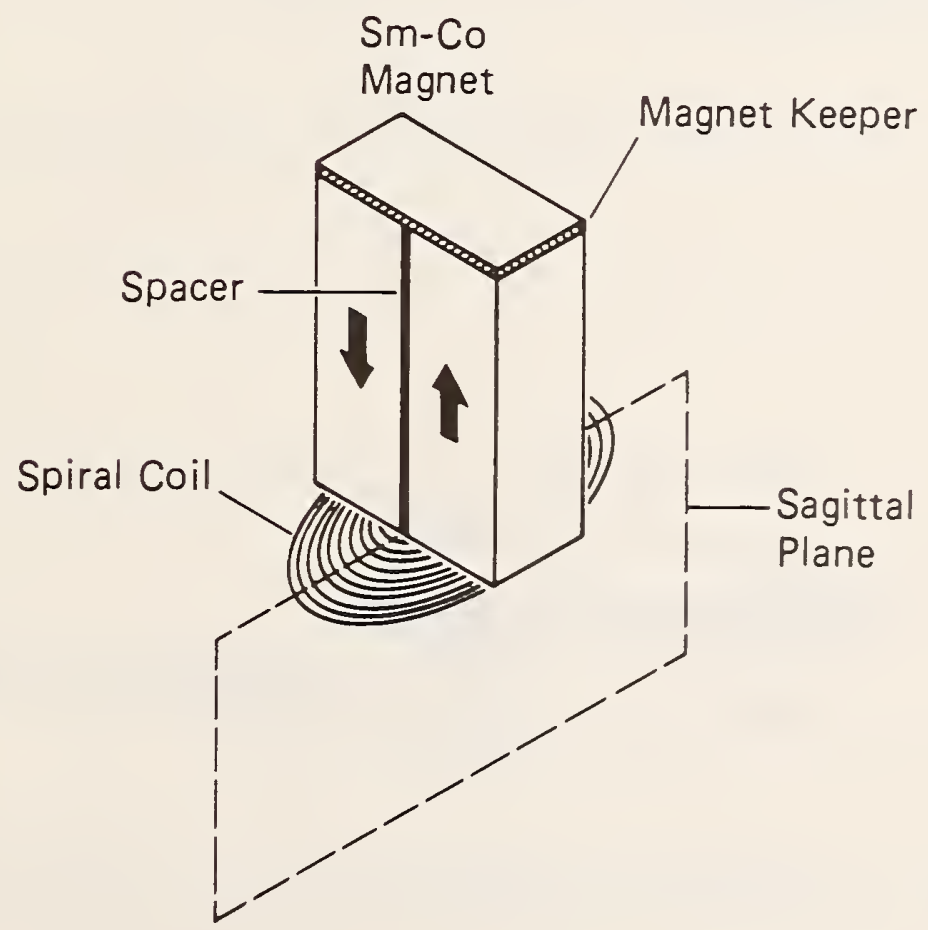

Fig. 4. Transducer construction. 


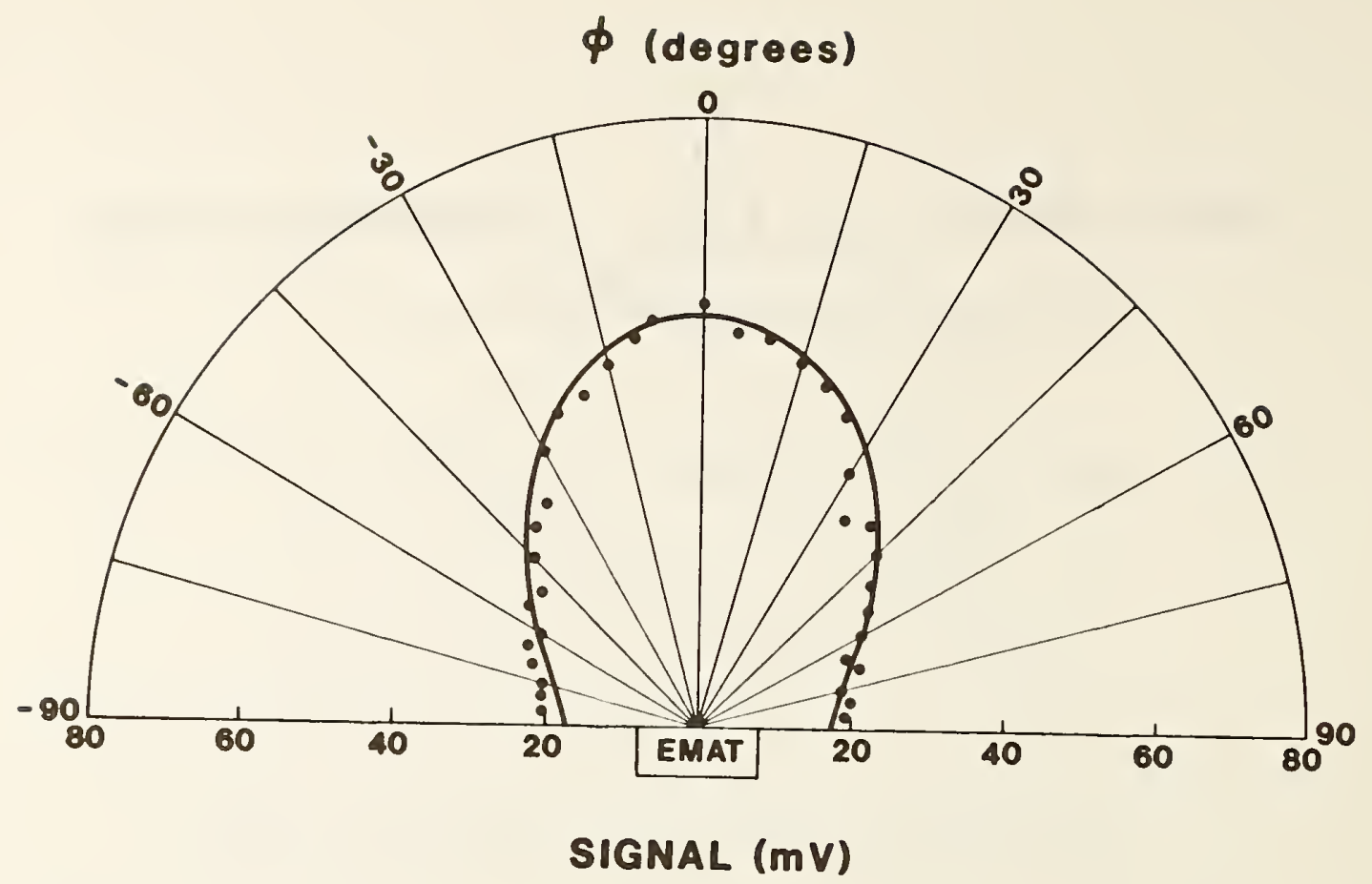

Fig. 5. Radiation pattern of transducer in fig. 4.

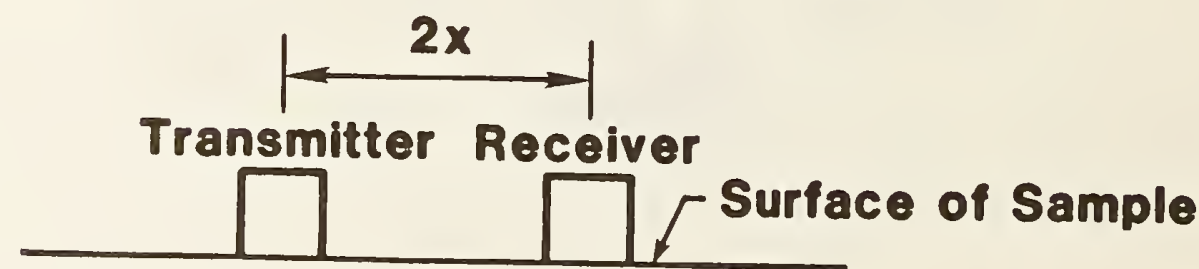

Fig. 6. Transducer configuration used in experiments. 


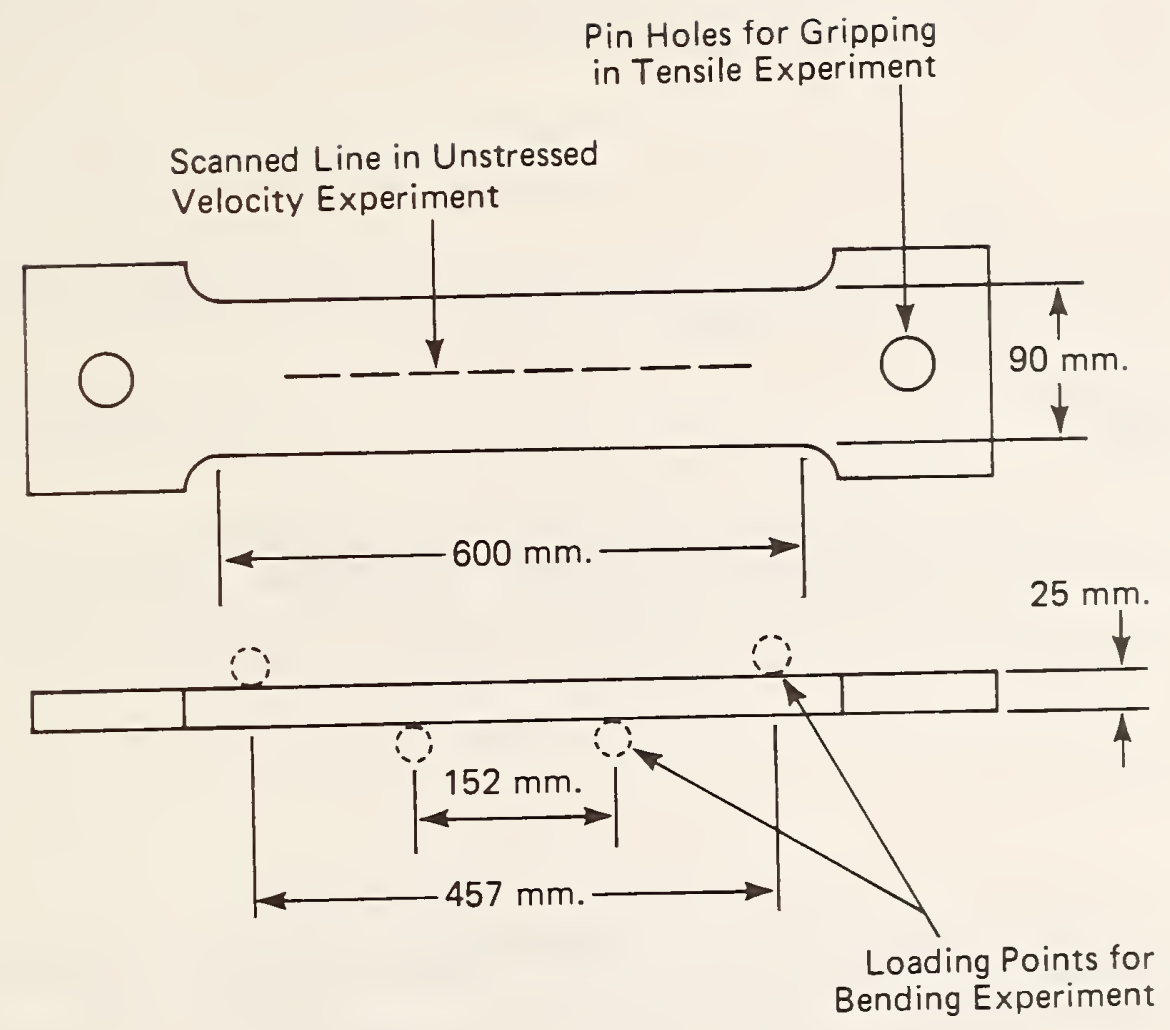

Fig. 7. Plate specimen used in experiments.

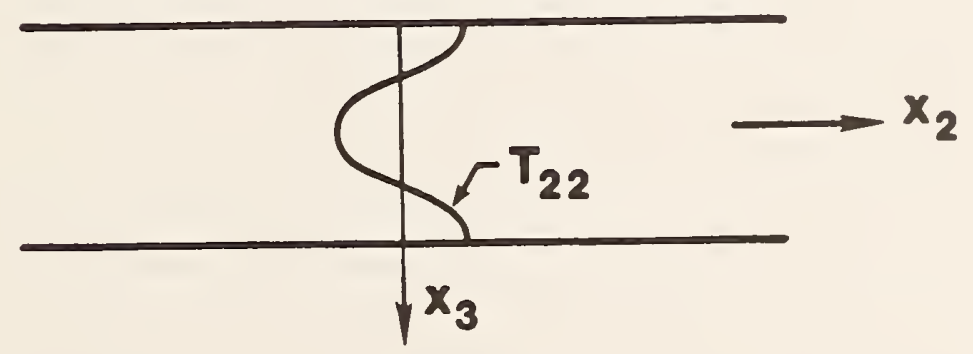

Fig. 8. Schematic of through-thickness variation of residual stress in a plate sample. 
NBS-114A (REV. $2-8 \mathrm{C}$ )

\begin{tabular}{|c|c|c|c|}
\hline $\begin{array}{c}\text { U.S. DEPT. OF COMM. } \\
\text { BIBLIOGRAPHIC DATA } \\
\text { SHEET (See instructions) }\end{array}$ & $\begin{array}{l}\text { 1. PUBLICATION OR } \\
\text { REPORT NO. } \\
\text { NBSIR } 84-3002\end{array}$ & 2. Performing Organ. Report No. & $\begin{array}{l}\text { 3. Publication Date } \\
\text { March }\end{array}$ \\
\hline
\end{tabular}

4. TITLE AND SUBTITLE

Residual-Stress Measurements Using Shear-Horizontal Waves from Electromagnetic

Acoustic Transducers

5. $A \cup T H O R(S)$

R. B. King and C. M. Fortunko

6. PERFORMING ORGANIZATION (If joint or other than NBS, see instructions)

7. Contract/Grant No.

NATIONAL BUREAU OF STANDARDS

DEPARTMENT OF COMMERCE

WASHINGTON, D.C. 20234

9. SPONSORING ORGANIZATION NAME AND COMPLETE ADDRESS (Street, City, State, ZIP)

U.S. Navy, David Taylor Naval Ship Research and Development Center, Annapolis, MD 2140

Office of Nondestructive Evaluation, National Bureau of Standards, Wash., D.C. 20234

10. SUPPLEMENTARY NOTES

Document describes a computer program; SF-185, FIPS Software Summary, is attached.

11. ABSTRACT (A 200-word or less factual summary of most significant information. If document includes a significant bibliography or literature survey, mention it here)

This collection of technical papers covers a two-year effort on a novel approach to measuring residual stresses using changes in ultrasonic wave velocities of horizontally polarized shear waves produced by electromagnetic-acoustic transducers. The initial three papers deal with the theoretical developments pointing to the measurement of in-plane residual stress as well as preliminary experimental verification. The later two papers generalize this theory and extend it to include surface residual stress measurement in an arbitrary plane; again, empirical validation is included.

12. KEY WORDS (Six to twelve entries: alphabetical order; capitalize only proper names; and separate key words by semicolons) acoustic; electromagnetic; nondestructive; residual stress; shear horizontal; velocity

13. AVAILABILITY

$[X]$ Unlimited

$\square$ For Official Distribution. Do Not Release to NTIS

PRINTED PAGES

$\square$ Order From Superintendent of Documents, U.S. Government Printing Office, Washington, D.C. 20402.

[X] Order From National Technical Information Service (NTIS), Springfield, VA. 2216I

15. Price

$\$ 10.00$ 



